\title{
An Efficient Inductor-less Dynamically Configured Interface Circuit for Piezoelectric Vibration Energy Harvesting
}

\author{
Sijun Du, Student Member, IEEE, Yu Jia, Member, IEEE, and Ashwin A. Seshia, Senior Member, IEEE
}

\begin{abstract}
Vibration energy harvesting based on piezoelectric materials is of interest in several applications such as in powering remote distributed wireless sensor nodes for structural health monitoring. Synchronized Switch Harvesting on Inductor (SSHI) and Synchronous Electric Charge Extraction (SECE) circuits show good power efficiency among reported power management circuits; however, limitations exist due to inductors employed, adaption of response to varying excitation levels and the Synchronized Switch Damping (SSD) effect. In this paper, an inductor-less dynamically configured interface circuit is proposed, which is able to configure the connection of two piezoelectric materials in parallel or in series by periodically evaluating the ambient excitation level. The proposed circuit is designed and fabricated in a $0.35 \mu \mathrm{m}$ HV CMOS process.The fabricated circuit is co-integrated with a piezoelectric bimorph energy harvester and the performance is experimentally validated. With a low power consumption $(0.5 \mu \mathrm{W})$, the measured results show that the proposed rectifier can provide a $4.5 \times$ boost in harvested energy compared to the conventional full-bridge rectifier without employing an inductor. It also shows a high power efficiency over a wide range of excitation levels and is less susceptible to SSD.
\end{abstract}

Index Terms-Energy harvesting, piezoelectric transducer, rectifier, power conditioning.

\section{INTRODUCTION}

$\mathbf{U}$ LTRA low power wireless sensors and sensor systems [1], [2] are of increasing interest in a variety of applications ranging from structural health monitoring to industrial process control [3], [4]. Compared to the wired methodologies, wireless devices provide many advantages, such as flexibility and ease of placing the sensors in locations that are not accessible by the wired counterparts. However, considerations of ultra low power are increasingly important for all wireless devices including sensors [5]. By minimizing the power consumption, researchers and engineers have attempted to extend battery lifetime and to avoid replacing or recharging batteries too frequently. While batteries have remained the primary energy sources due to their energy density, in certain sensing contexts requiring the operation of sensors and sensor systems over a significant period of time [6], including implantable biomedical electronic devices [7] and tire pressure sensors [8], battery usage may be both impractical and add extra cost due to the requirements for periodic re-charging and/or replacement [9]. In order to address this challenge and extend

Sijun Du and Ashwin A. Seshia are with the Department of Engineering, University of Cambridge, Cambridge, CB2 1PZ, U.K. (e-mail: sd672@cam.ac.uk; aas41@cam.ac.uk)

$\mathrm{Yu}$ Jia is with Department of Mechanical Engineering, University of Chester, Chester, CH2 4NU, U.K. (e-mail: y.jia@chester.ac.uk) the operational lifetime of wireless sensors, there has been an emerging research interest to harvest energy from environmental kinetic vibration [10], [11].

Among all vibration energy harvesters (VEH), piezoelectric materials are widely used due to their scalability and compatibility with integrated circuit technologies [12] compared to their electrostatic [13] and electromagnetic counterparts [14]. The piezoelectric transducer (PT) has to be designed to not only produce as much raw power as possible, but the interface circuit also needs to be able to extract most of power from the PT with very low power consumption. Full-bridge rectifiers are widely used in commercial energy harvesting systems due to their simplicity and stability; however, they set high threshold voltages for the generated energy to be extracted by the circuit. While vibrating at or close to its resonance, a PT can be modeled as a current source $I_{P}$ connected in parallel with a plate capacitor $C_{P}$ and a resistor $R_{P}$. Fig. 1 shows the full-bridge rectifier connected with a PT and the associated waveforms. In order to transfer the generated energy from the PT to the storage capacitor $C_{S}$, the voltage across the PT $V_{\text {piezo }}$ should attain $V_{S}+2 V_{D}$ or $-\left(V_{S}+2 V_{D}\right)$. Hence the energy used to charge the internal capacitor $C_{P}$ from $V_{S}+2 V_{D}$ to $-\left(V_{S}+2 V_{D}\right)$ (or vice-versa) is wasted.

In order to increase the power efficiency of a VEH system, most of active rectifiers seek to develop a mechanism to minimize the energy wasted in charging $C_{P}$. An SSHI (Synchronized Switch Harvesting on Inductor) rectifier was presented in [15] to employ an inductor to flip the voltage $V_{\text {piezo }}$ at zerocrossing points of $I_{P}$. Chip and board level measurements of SSHI rectifiers have been previously implemented in [16], [17] to demonstrate their high power efficiency. Other synchronized switch interfaces, such as Synchronous Electric Charge Extraction (SECE), are also widely used for high-efficiency circuits [18].

Although SSHI and SECE rectifiers can transfer most of charge to a storage capacitor at specific conditions, they have a few main drawbacks that need to be mentioned. First, SSHI and SECE circuits require inductors, which must be implemented off-chip to achieve good performance and such an inductor can be the main factor in increasing the overall volume of the energy harvesting system. In addition, SSHI circuits can only achieve high efficiency at a limited range of excitation levels. This limits the overall performance of the circuit in real-world implementations, where the excitation level varies with time unpredictably in a wide range. Although this is not an issue for an SECE circuit due to its different ar- 


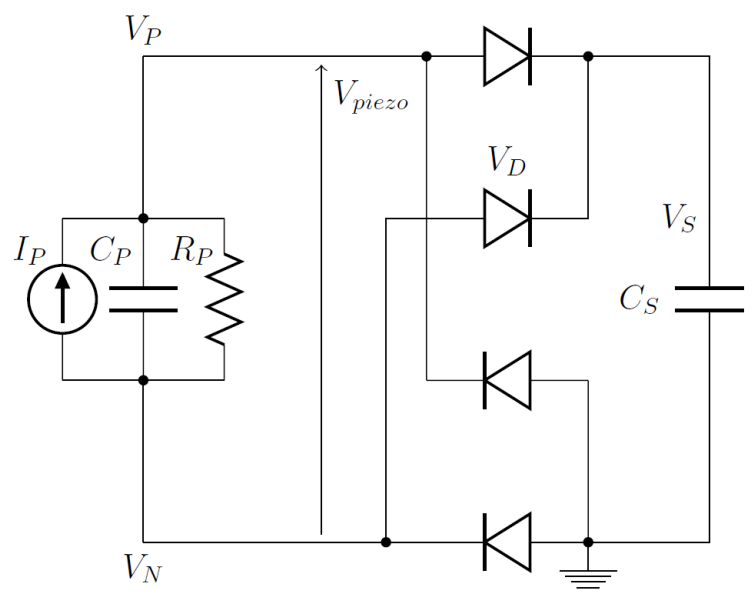

(a) Full-bridge rectifier

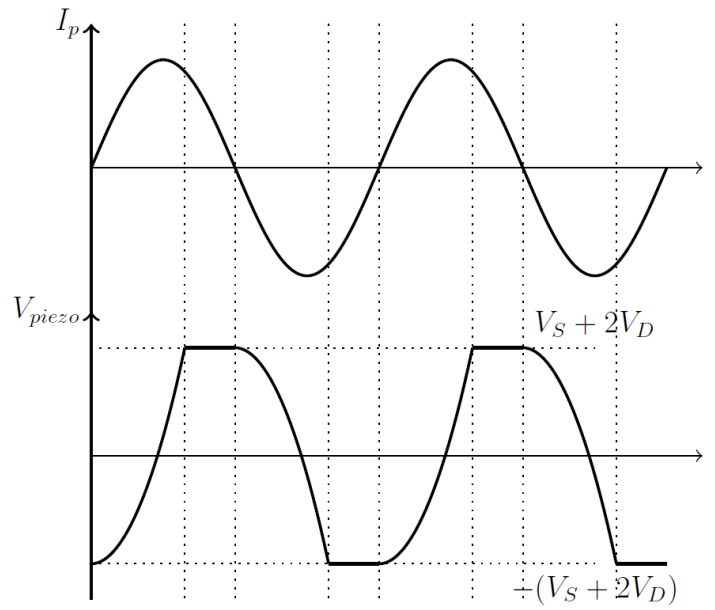

(b) $I_{p}$ and $V_{\text {piezo }}$ waveform

Fig. 1: Full-bridge rectifier for piezoelectric VEH and the associated waveforms

chitecture to extract energy, it required more complex circuits to be implemented compared to other circuits. Furthermore, SSHI and SECE can only provide higher performance than simple full-bridge rectifiers for weakly coupled piezoelectric transducers due to the synchronized switch damping effect. If the the coupling is strong and the PT vibrates at resonance, the periodic current pulses applied to invert (for SSHI) or extract (for SECE) charge on a PT result in an electrical actuation that opposes the vibration, which is known as Synchronized Switch Damping (SSD) [19], [20]. Due to the relatively strong nonlinear damping introduced, this principle has also been used for wave reflection/transmission reduction [21], where an architecture similar to SSHI was used to perform the charge inversion to increase the electrical actuation. This negative force feedback is basically introduced by the first harmonic of the current pulses. If the current pulses are lower and wider (lower amplitude and lower first harmonic frequency), the SSD becomes less significant and synchronized switch circuits can thus transfer charge. All of the above limitations introduced by inductors, real-world wide range excitation levels and SSD effect result in the SSHI and SECE rectifiers achieving acceptable performance only in a limited operating range.

This paper proposes a fully integrated CMOS interface circuit interfaced to a bimorph PT to automatically switch the connection of the two PTs to increase output power based on the amplitude of the input excitation, thereby enabling a significant improvement in power extraction efficiency for the immediate electrical interface. With the proposed circuit, the two PTs are connected in parallel or in series according to the environmental excitation level by periodically evaluating the excitation amplitude. As compared to the SSHI or SECE rectifiers, the proposed circuit does not employ any inductor, which significantly decrease the expected overall volume of the system, especially for MEMS low-volume energy harvesters. In addition, dynamically switching between parallel and series configurations allows the energy harvester to achieve a high power efficiency over a wide range of input excitation amplitudes. In terms of the SSD problems for SSHI and SECE rectifiers, the proposed circuit enables shifting between different configurations instead of performing synchronized charge inversion or extraction. Hence it avoids introducing negative force feedback and it is less subject to the SSD introduced by the circuit, which extends the range over which the circuit can operate efficiently.

This paper consists of six sections presenting the proposed circuit covering modeling and experimental validation. In the next section, parallel and series connections of two PTs are theoretically studied to identify the conditions when one configuration is working better than the other one. Identifying the switching condition between parallel and series models is very important to implement the associated algorithms in designing the circuit. The third section gives an overall description of the proposed interface circuit and the fourth section provides details in circuit implementations of each functional block of the system. The fabricated chip is experimentally evaluated in the fifth section and the final section provides a summary and conclusion.

\section{MODELING OF PARALLEL AND SERIES CONFIGURATIONS}

In this section, theoretical models are developed to compare the performance between parallel and series connections of a bimorph cantilever. In order to compare the performance, there are two methods to evaluate the output power from the both models. One way is to change excitation amplitude (corresponding to voltage $V_{\text {piezo }}$ ) with a fixed $V_{S}$; another way is to change the voltage $V_{S}$ for a fixed excitation amplitude. The proposed rectifier aims to choose an appropriate connection type according to both excitation amplitude and $V_{S}$ value to maximize output power.

\section{A. Parallel model}

As the two piezoelectric transducers (PT) are located on the both sides of a single bimorph cantilever, they have exactly the same frequencies, amplitudes and phases. While the two PTs are connected in parallel, the parallel model can be considered 


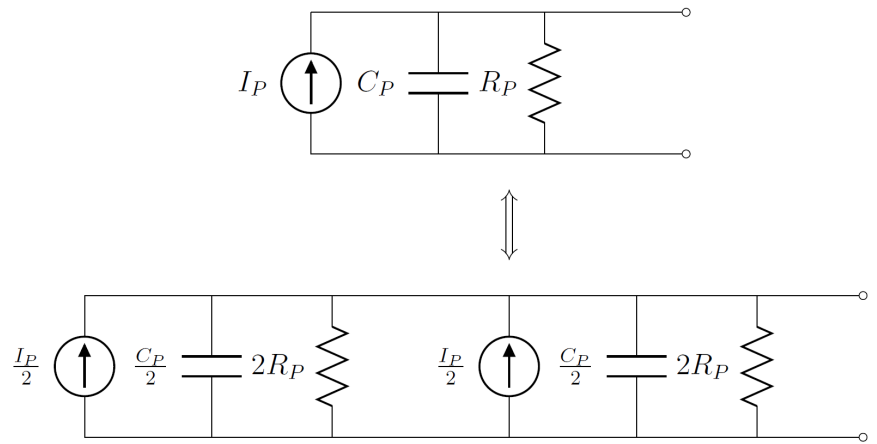

Fig. 2: A monolithic PT (top) and two PTs connected in parallel (bottom)

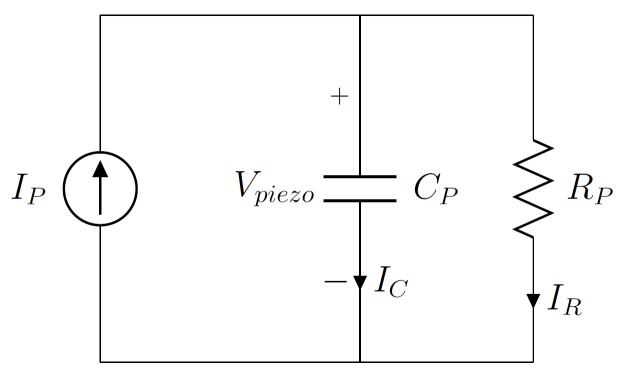

Fig. 3: Current flow in a piezoelectric generator

as a $2 \times$ larger monolithic PT with frequency, amplitude and phase unchanged, see Fig. 2. Assuming the excitation is sinusoidal, the current source, capacitor and resistor for the resulting parallel model can be written as $I_{P}=I_{0} \sin 2 \pi f_{P} t$, $C_{P}$ and $R_{P}$. Hence, the corresponding parameters for one single PT are $\frac{1}{2} I_{P}, \frac{1}{2} C_{P}$ and $2 R_{P}$. For the parallel model, the total generated charge in a half cycle $T / 2$ should first be calculated and can be written as:

$$
Q_{\text {total }}=\int_{0}^{\frac{T}{2}} I_{0} \sin \omega t \mathrm{~d} t=\frac{2 I_{0}}{\omega}
$$

Before the full-bridge rectifier becomes conducting, the current from $I_{P}$ is split into two parts inside the piezoelectric harvester, $I_{C}$ and $I_{R}$ flowing through the capacitor and resistor respectively (see Fig. 3). As the rectifier is not yet conducting in this case, the PT can be regarded as operating in an opencircuit. Hence, the charge flowing into the capacitor $C_{P}$ can be written as:

$$
Q_{C}(j \omega)=Q_{\text {total }} \frac{I_{C}}{I_{P}}(j \omega)=\frac{2 j I_{0} R_{P} C_{P}}{1+j \omega R_{P} C_{P}}
$$

Besides the charge flowing into $C_{P}$ to form the voltage $V_{\text {piezo }}$, the rest of the charge is dissipated by the resistor $R_{P}$. According to the formula $V=Q / C$, the open-circuit peakto-peak voltage $V_{p p(o p e n)}$ is expressed as:

$$
\begin{aligned}
V_{p p(\text { open })}=\left|\frac{Q_{C}(j \omega)}{C_{P}}\right| & =\left|\frac{2 j I_{0} R_{P}}{1+j \omega R_{P} C_{P}}\right| \\
& =\frac{2 I_{0} R_{P}}{\sqrt{1+\omega^{2} R_{P}^{2} C_{P}^{2}}}
\end{aligned}
$$

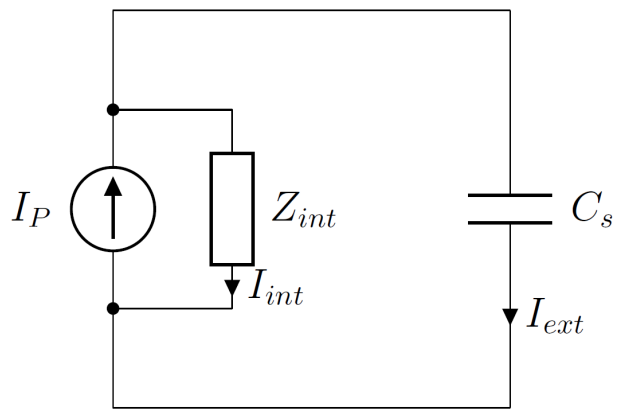

Fig. 4: Current flow while charging $C_{s}$ - parallel model

In order to be able to charge the capacitor $C_{S}$, the voltage $V_{p p \text { (open) }}$ should be greater than the threshold $V_{T H}=2\left(V_{S}+\right.$ $\left.2 V_{D}\right)$. Hence, the condition for the rectifier to start transferring charge from the PT to $C_{S}$ is:

$$
\begin{aligned}
V_{\text {pp(open })} & >2\left(V_{S}+2 V_{D}\right) \\
\Rightarrow \frac{I_{0} R_{P}}{\sqrt{1+\omega^{2} R_{P}^{2} C_{P}^{2}}} & >V_{S}+2 V_{D}
\end{aligned}
$$

Assuming $V_{S}=2 \mathrm{~V}$ and $V_{D}=0.3 \mathrm{~V}$, the condition for commencing transferring energy for the parallel model is $V_{p p(\text { open })}>2\left(V_{S}+2 V_{D}\right)=5.2 \mathrm{~V}$. In order to compare the performance between parallel and series models, this condition is assumed to be always satisfied. The charge flowing into $C_{P}$ is expressed in (2). After a part of charge is wasted for charging $C_{P}, V_{\text {piezo }}$ equals to $V_{S}+2 V_{D}$ (or $-\left(V_{S}+2 V_{D}\right)$ ) and the rectifier becomes conducting. The wasted charge can be expressed as: $Q_{\text {wasted }}=2 C_{P}\left(V_{S}+2 V_{D}\right)$. Therefore, the charge going through the rectifier is the difference between $Q_{C}$ and $Q_{\text {wasted }}$ :

$$
\begin{aligned}
Q_{\text {remain }}(j \omega) & =Q_{C}(j \omega)-Q_{\text {wasted }} \\
& =2 C_{P}\left(\frac{j I_{0} R_{P}}{1+j \omega R_{P} C_{P}}-\left(V_{S}+2 V_{D}\right)\right)
\end{aligned}
$$

After the rectifier becomes conducting, the voltage $V_{\text {piezo }}$ attains the threshold and the equivalent circuit transforms to a harvester in parallel with $C_{S}$ as shown in Fig. 4. The internal impedance of the piezoelectric harvester is the value that $C_{P}$ and $R_{P}$ in parallel, expressed as $Z_{i n t}(j \omega)=\frac{1}{j \omega C_{P}} / / R_{P}=$ $\frac{R_{P}}{1+j \omega R_{P} C_{P}}$. Hence, the charge flowing into $C_{S}$ can be written as:

$$
\begin{aligned}
& Q_{S}(j \omega)=Q_{\text {remain }} \frac{Z_{\text {int }}}{Z_{\text {int }}+\frac{1}{j \omega C_{S}}} \\
& =\frac{2 j \omega R_{P} C_{P} C_{S}}{1+j \omega R_{P}\left(C_{P}+C_{S}\right)}\left(\frac{j I_{0} R_{P}}{1+j \omega R_{P} C_{P}}-\left(V_{S}+2 V_{D}\right)\right)
\end{aligned}
$$

The capacitor $C_{S}$ at the output of the rectifier is usually chosen at a value much higher than the PT internal capacitor $C_{P}\left(C_{S} \gg C_{P}\right)$, so that $V_{S}$ can keep increasing steadily. In addition, as $R_{P}$ is usually between hundreds of $\mathrm{k} \Omega$ and several $\mathrm{M} \Omega$, hence $\omega R_{P} C_{S} \gg 1$. Therefore, (6) can be approximately written as: 


$$
\begin{aligned}
Q_{S} & \approx 2 C_{P}\left(\frac{I_{0} R_{P}}{\sqrt{1+\omega^{2} R_{P}^{2} C_{P}^{2}}}-\left(V_{S}+2 V_{D}\right)\right) \\
& =2 C_{P}\left(\frac{V_{p p(\text { open })}}{2}-\left(V_{S}+2 V_{D}\right)\right.
\end{aligned}
$$

So that the voltage increase in $C_{S}$ for the parallel model in a half $I_{P}$ cycle is expressed as (where the subscript "//" means "parallel"):

$$
\Delta V_{S / /}=\frac{Q_{S}}{C_{S}}=\frac{C_{P}}{C_{S}}\left(V_{p p(\text { open })}-2\left(V_{S}+2 V_{D}\right)\right)
$$

\section{B. Series model}

For the two PTs connected in series, the calculation starts with considering a single harvester, for which the internal current flow is similar to that shown in figure 4 and $V_{\text {piezo } 1}$ is the voltage generated by one single source. As there are two PTs connected in series, the total voltage is $V_{\text {piezo }}=$ $\sum_{i=1}^{2} V_{\text {piezoi }}=2 V_{\text {piezo1 }}$. As the condition to charge $C_{S}$ is $V_{\text {piezo }}>2\left(V_{S}+2 V_{D}\right)$, hence this condition for each individual source is $V_{\text {piezo } 1}>V_{S}+2 V_{D}$. It can be seen that the threshold voltage is now lowered by two times compared to the parallel model so that harvester is more likely to start operating at lower excitation levels. Hence, the charge flowing into $C_{P 1}$ in a half cycle is:

$$
Q_{\frac{T}{2} 1}(j \omega)=\int_{0}^{\frac{T}{2}} I_{p 1} \frac{R_{P 1}}{R_{P 1}+\frac{1}{j \omega C_{p 1}}}=\frac{I_{0} R_{P} C_{P}}{1+j \omega R_{P} C_{P}}
$$

The wasted charge for dis-charging and charging in one source in a half cycle is:

$$
Q_{\text {wasted } 1}=C_{p 1}\left(V_{S}+2 V_{D}\right)=\frac{C_{p}}{2}\left(V_{S}+2 V_{D}\right)
$$

Before the condition $V_{\text {piezo1 }}>V_{S}+2 V_{D}$ is met, the harvester is disconnected from $C_{S}$ (as the diodes in the rectifier are not conducting). Once the $V_{\text {piezo } 1}>V_{S}+2 V_{D}$ is satisfied, all of the sources are connected together with $C_{S}$ in series. At this time, $C_{S}$ starts to be charged and the remaining charge for each single source that can be used for charging is:

$$
\begin{aligned}
& Q_{\text {remain } 1}(j \omega)=Q_{\frac{T}{2} 1}(j \omega)-Q_{\text {wasted } 1} \\
& \quad=C_{P}\left(\frac{I_{0} R_{P}}{1+j \omega R_{P} C_{P}}-\frac{V_{S}+2 V_{D}}{2}\right)
\end{aligned}
$$

As only one harvester is considered, superposition theory can be used to turn off the current source of the other harvester. While the rectifier is conducting, the equivalent circuit for one single source is shown in figure 5. As the total internal capacitance and resistance for the parallel model are $C_{P}$ and $R_{P}$, these values for one single PT becomes $C_{P} / 2$ and $2 R_{P}$. Hence, the internal impedance for one PT is $Z_{i n t 1}(j \omega)=\frac{2}{j \omega C_{P}} / / 2 R_{P}=\frac{2 R_{P}}{1+j \omega R_{P} C_{P}}$. Therefore, the ratio between the $I_{e x t}$ and $I_{i n t}$ for each source being studied is:

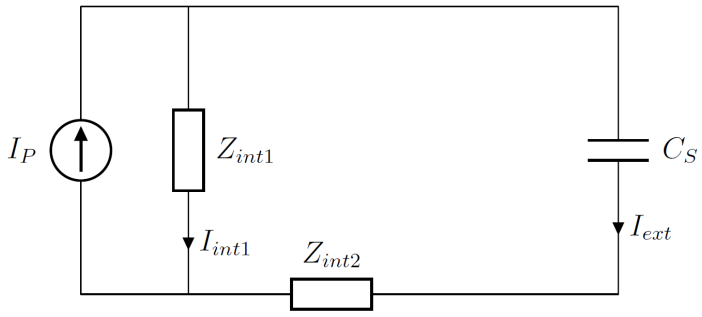

Fig. 5: Equivalent circuit for two PTs connected in series for charging $C_{S}$

$$
\begin{aligned}
& \frac{I_{\text {ext }}}{I_{\text {int }}}=\left|\frac{Z_{\text {int } 1}}{Z_{\text {int } 1}+Z_{\text {int } 2}+\frac{1}{j \omega C_{S}}}\right| \approx \frac{1}{2} \\
& \left(\text { as } C_{S} \gg C_{P} \text { and } Z_{\text {int } 1}=Z_{\text {int } 2}\right)
\end{aligned}
$$

Therefore, the total charge that flows into $C_{S}$ from one single source is:

$Q_{S 1}=\left|\frac{1}{2} Q_{l e f t 1}(j \omega)\right|=\frac{C_{P}}{2}\left(\frac{I_{0} R_{P}}{\sqrt{1+\omega^{2} R_{P}^{2} C_{P}^{2}}}-\frac{V_{S}+2 V_{D}}{2}\right)$

With consideration of the other PT, the total charge that flows into $C_{S}$ is (the subscript "+" in the expression represents series connection):

$$
Q_{S+}=2 Q_{S 1}=C_{P}\left(\frac{I_{0} R_{P}}{\sqrt{1+\omega^{2} R_{P}^{2} C_{P}^{2}}}-\frac{V_{S}+2 V_{D}}{2}\right)
$$

The voltage increase in $C_{S}$ is:

$$
\Delta V_{S+}=\frac{Q_{S+}}{C_{S}}=\frac{C_{P}}{C_{S}}\left(\frac{V_{p p(o p e n)}}{2}-\frac{V_{S}+2 V_{D}}{2}\right)
$$

\section{Performance comparison and proposed scheme}

The voltage increase values in $V_{S}$ for both parallel and series models are expressed in (8) and (15). As these are the voltage variation in a half $I_{P}$ period, the output power for both models can be calculated by dividing the increased energy stored in $C_{S}$ by the half period:

$P=\frac{\frac{1}{2} C_{S}\left(\left(V_{S}+\Delta V_{S}\right)^{2}-V_{S}^{2}\right)}{T / 2}=f_{P} C_{S}\left(\left(V_{S}+\Delta V_{S}\right)^{2}-V_{S}^{2}\right)$

where $\Delta V_{S}$ can be either $\Delta V_{S / /}$ expressed in (8) for the parallel model or $\Delta V_{S+}$ expressed in (15) for the series model. With given diodes (fixed $V_{D}$ ), there are two variables in (16): $V_{p p(o p e n)}$ and $V_{S}$. The performance of both models can be compared while fixing one of these variables and varying the other one. Fig. 6 shows the theoretical comparison of parallel and series models in function of excitation amplitude (Fig. 6a) and in function of $V_{S}$ (Fig. 6b), where the diode voltage drop is set as $V_{D}=0.3 \mathrm{~V}$. It can be seen that each model has an optimal operation range compared to the other model. Hence, it is useful to find the condition when the parallel model 


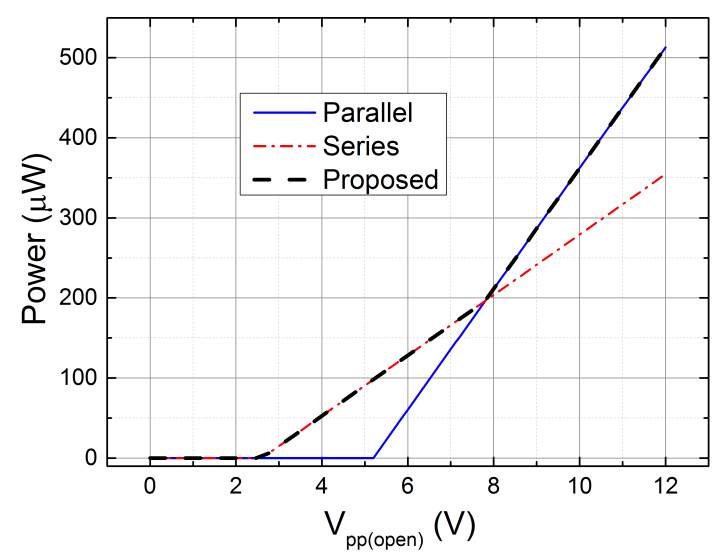

(a) Output power in function of excitation level with fixed $V_{S}=2 \mathrm{~V}$

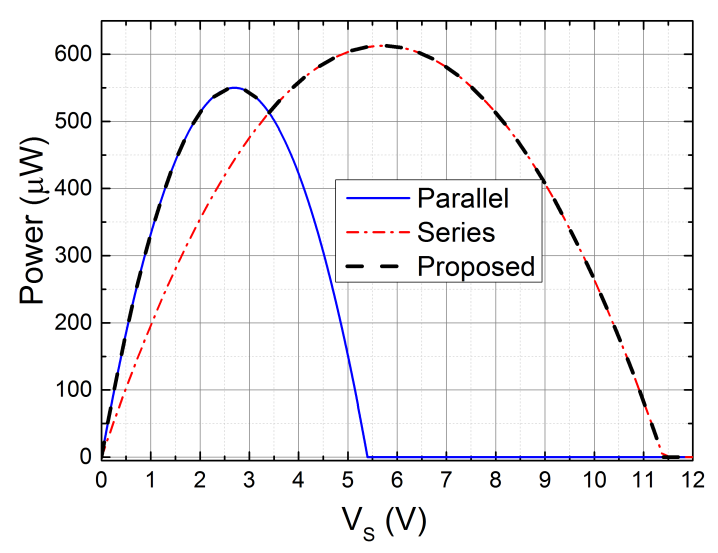

(b) Output power in function of $V_{S}$ with fixed excitation level of $V_{p p(\text { open })}=12 \mathrm{~V}$ (acceleration: $\left.8.0 \mathrm{~g}\right)$

Fig. 6: Theoretical output power for parallel model, series model and proposed model (diode voltage drop set as $V_{D}=0.3 \mathrm{~V}$ )

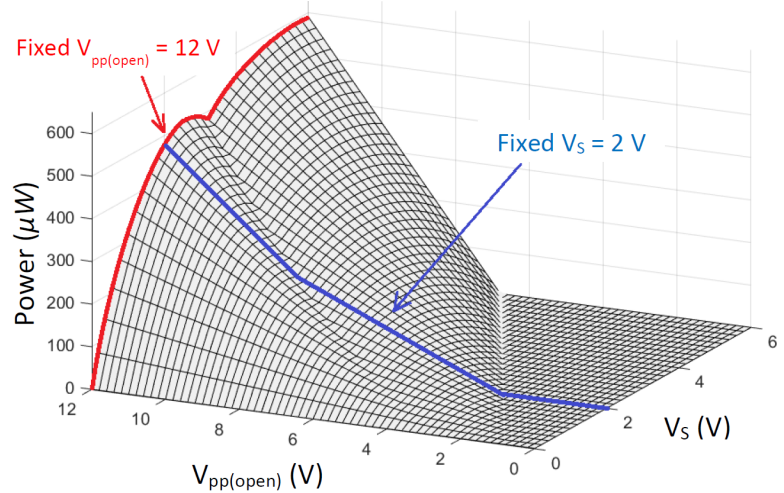

Fig. 7: 3-D surface plot of theoretical output power in function of $V_{p p(o p e n)}$ and $V_{S}$

outputs higher power than the series model. This condition can be found by setting $\Delta V_{S / /}>\Delta V_{S+}$ :

$$
\begin{aligned}
\frac{C_{P}}{C_{S}}\left(V_{p p(\text { open })}-2\left(V_{S}+2 V_{D}\right)\right. & >\frac{C_{P}}{C_{S}}\left(\frac{V_{p p(\text { open })}}{2}-\frac{V_{S}+2 V_{D}}{2}\right) \\
\Rightarrow V_{p p(\text { open })} & >3\left(V_{S}+2 V_{D}\right)
\end{aligned}
$$

It should be noticed that the value $V_{p p(o p e n)}$ is the voltage while the two PTs are connected in parallel because this value doubles for the series model. The inequality in (17) shows the condition that the parallel model can generate more output power than the series model. Fig. 6 shows that the output power difference from the two models can be significant in some cases. Therefore, making a good choice between parallel and series connections in a specific condition can increase the output power and the operational excitation range. The proposed interface circuit in this paper is able to check the condition in (17) periodically and connect the two PTs in parallel if the condition is satisfied; otherwise, in series. The expected output power of the proposed circuit is shown in dash curves.
According to (8), (15) and (16), the output power while using the proposed circuit can be expressed as:

$$
\begin{aligned}
& P=f_{P} C_{S}\left(\left(V_{S}+\Delta V_{S}\right)^{2}-V_{S}^{2}\right) \quad \text { where } \\
& \Delta V_{S}=\left\{\begin{array}{c}
\frac{C_{P}}{C_{S}}\left(V_{p p(\text { open })}-2\left(V_{S}+2 V_{D}\right)\right) \\
\quad \text { if } V_{p p(\text { open })} \geq 3\left(V_{S}+2 V_{D}\right) \\
\frac{C_{P}}{C_{S}}\left(\frac{V_{p p(\text { open })}}{2}-\frac{V_{S}+2 V_{D}}{2}\right) \\
\quad \text { if }\left(V_{S}+2 V_{D}\right) \leq V_{p p(\text { open })}<3\left(V_{S}+2 V_{D}\right) \\
0 \quad \\
\quad \text { if } V_{p p(\text { open })}<\left(V_{S}+2 V_{D}\right)
\end{array}\right.
\end{aligned}
$$

If both the two variables $V_{p p(o p e n)}$ and $V_{S}$ are swept $0 \mathrm{~V} \rightarrow$ $12 \mathrm{~V}$ and $0 \mathrm{~V} \rightarrow 6 \mathrm{~V}$ respectively, a three dimensional surface plot of output power can be plotted, which is shown in Fig. 7. Planes of $V_{S}=2 \mathrm{~V}$ (corresponding to Fig. 6a) and $V_{p p(o p e n)}=$ $12 \mathrm{~V}$ (corresponding to Fig. 6b) are highlighted in this figure. It can be seen that higher $V_{S}$ requires higher $V_{p p(\text { open })}$ to start transferring energy to the storage capacitor. With a fixed $V_{S}$, the series model is able to output much higher power than the parallel model in low excitation levels. While $V_{p p(o p e n)}$ goes higher, this difference becomes smaller but two peak power points allows a high output power in a wide range of $V_{S}$.

\section{Proposed interface CiRCUIT}

Fig. 8 shows the implementation of the proposed interface circuit between two PTs (a bimorph cantilever is used in this implementation) and a full-bridge rectifier. An off-chip voltage regulator is employed to provide a stable power supply $V_{D D}=$ $1.5 \mathrm{~V}$ to power the interface circuit itself and any possible future load electronics. The two PTs are the two piezoelectric layers on a bimorph cantilever, so that they have the same frequencies, amplitudes and phases. The system architecture of the proposed circuit is also shown in the figure, which consists of a connection switching block, a power management 


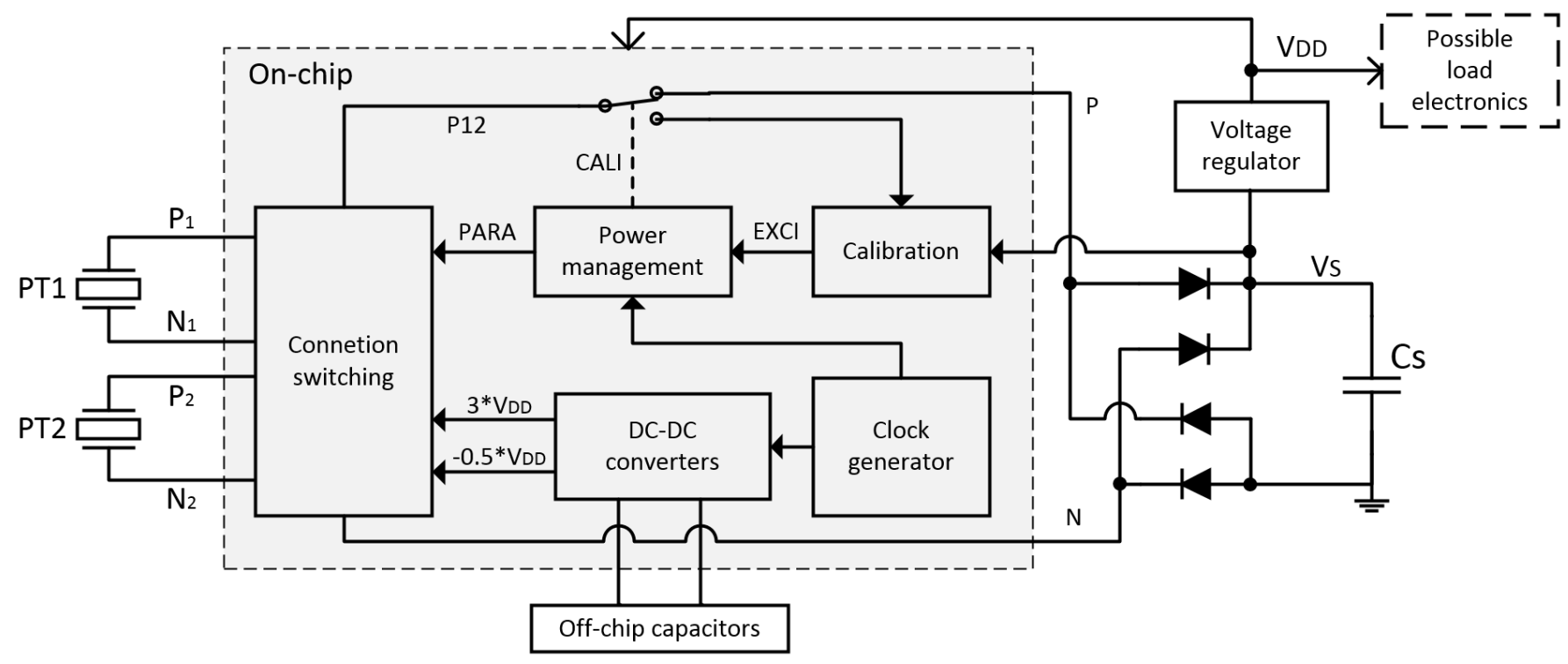

Fig. 8: Architecture of proposed system

block, a calibration block, an internal clock generator and switched-capacitor (SC) DC-DC converters. The connection switching block enables parallel or series configurations of the PTs according to the signal $P A R A$ (high for parallel and low for series). The power management block is a digital block that sets the system in the "sleep mode" for most of the time and in "calibration mode" to evaluate excitation amplitudes and re-connect the two PTs. The calibration block performs the algorithm to check the condition in (17) and generates EXCI pulses if the condition is satisfied. The signal EXCI (short for 'excitation') indicates that the environmental excitation is high enough to make the circuit choosing parallel connection. The DC-DC converters aim to generate a high voltage level and a negative voltage level to overdrive the gates of analog switches in the connection switching block.

While the system is in "sleep mode", the calibration block is powered OFF to minimize the overall power consumption. The duration of the "sleep mode" is controlled by a digital counter in the "power management block" driven by an internally generated clock signal. This counting time can be externally set. While the "sleep mode" ends, the system goes into "calibration mode". In this mode, the connection is forced to be parallel with a high $P A R A$ signal and the node $P_{12}$ is disconnected from the node $P$ by signal $C A L I$ (short for 'calibration'); because the value $V_{p p \text { (open) }}$ in (17) requires that the two PTs are connected in parallel and in an open-circuit (not connected to the diodes). In this mode, the voltage at node $P_{12}$ and the voltage $V_{S}$ are used for comparison in an algorithm corresponding to the condition in (17). If the excitation level is high to satisfy the condition, $E X C I$ pulses will be generated to the power management block, which gives a final decision on the signal $P A R A$ and the "calibration mode" finishes.

\section{CiRCUIT IMPLEMENTATIONS}

This section describes the circuit implementations of the proposed connection auto-switching interface circuit as a

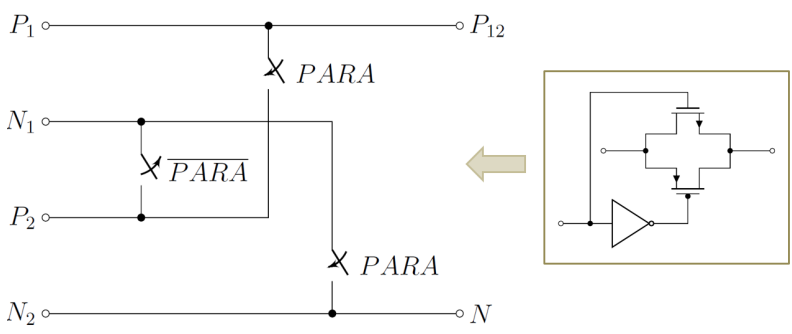

Fig. 9: Parallel-series connection switching circuitry with CMOS analogue switches

CMOS circuit. Some key blocks shown in Fig. 8 are presented in this section with circuit diagrams and relevant calculations.

\section{A. Parallel-series connection switching block}

The parallel-series connection switching circuitry utilizes three CMOS switches, as shown in Fig. 9. The nodes $P_{1}, N_{1}$, $P_{2}$ and $N_{2}$ are the electrodes of the two PTs. The node $N$ is one of the inputs of full-bridge rectifier. The node $P_{12}$ is connected to the other input of the rectifier while the system is in "sleep mode" and to the calibration block in "calibration mode", as shown in Fig. 8. In order to make sure that the switches are fully switched $\mathrm{ON}$ and $\mathrm{OFF}$ for relevant $P A R A$ signals, the gate driving voltage of $P A R A$ should fully cover the voltage ranges of all the six nodes in the figure. According to Fig. 1, the voltages of $V_{P}$ and $V_{N}$ are between $-V_{D}$ and $V_{S}+V_{D}$. Hence, the low level of signal PARA should be lower than $-V_{D}$ and its high level should be higher than $V_{S}+$ $V_{D}$. In this implementation, voltage levels of $V_{s u b}=-0.75 \mathrm{~V}$ and $V_{D D A}=4.5 \mathrm{~V}$ are chosen to drive the switches. The $\mathrm{N}$-channel MOSFETs used in the switches are isolated highvoltage transistors with a negative bulk voltage $V_{\text {sub }}$ and the bulk voltage of the P-channel MOSFETs is $V_{D D A}$.

As the signal $P A R A$ is generated from the power management block, which is a digital block, the voltage levels of 


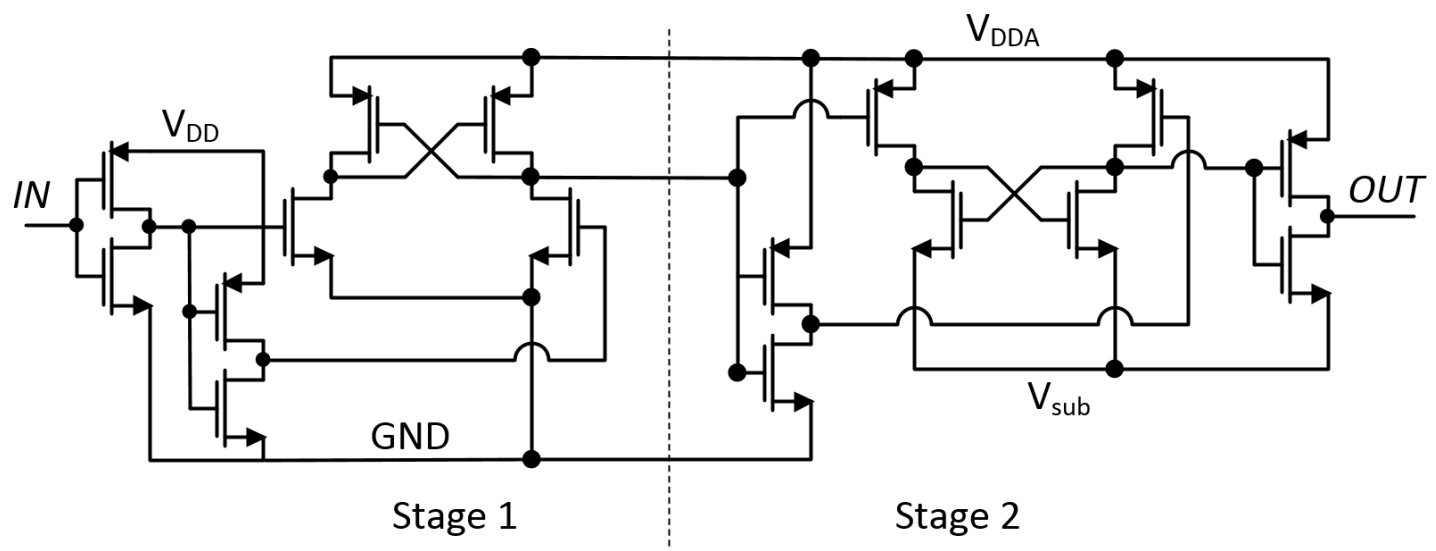

Fig. 10: Two-stage level-up shifter

$P A R A$ are $G N D$ and $V_{D D}$, where $V_{D D}=1.5 \mathrm{~V}$ is used in this implementation. Hence, before the signal $P A R A$ from the power management block can be used to drive the switches, a level-up shifter is needed to shift the voltage level $0 \mathrm{~V}$ to $-0.75 \mathrm{~V}$ and $1.5 \mathrm{~V}$ to $4.5 \mathrm{~V}$. The reason for choosing $-0.75 \mathrm{~V}$ and $4.5 \mathrm{~V}$ as the most negative and positive voltage levels in the circuit is due to the maximum allowed voltages for the selected transistors in the HV CMOS process used in this implementation. The absolute maximum allowed voltages $V_{G S}$ and $V_{D S}$ for these transistors are $5.5 \mathrm{~V}$ and the oxide breakdown voltage is $7 \mathrm{~V}$. Hence, choosing $-0.75 \mathrm{~V}$ and $4.5 \mathrm{~V}$ voltage levels makes a maximum $5.25 \mathrm{~V}$ voltage difference, which makes sure all the transistors operating safely. Fig. 10 shows a two-stage level-up shifter to shift the high level of the input signal to a higher voltage and the low level to a lower voltage. The different voltage levels shown in the figure are $G N D=0 \mathrm{~V}, V_{D D}=1.5 \mathrm{~V}, V_{D D A}=4.5 \mathrm{~V}$ and $V_{s u b}=-0.75 \mathrm{~V}$. The first stage employs a cross-coupled PMOS load and it aims to shift logic voltage levels from $[0 \mathrm{~V}$, $1.5 \mathrm{~V}]$ to $[0 \mathrm{~V}, 4.5 \mathrm{~V}]$. The second stage employs a crosscoupled NMOS load to further shift logic levels from $[0 \mathrm{~V}$, $4.5 \mathrm{~V}]$ to $[-0.75 \mathrm{~V}, 4.5 \mathrm{~V}]$. The typical quiescent current at room temperature for supply $V_{D D A}$ is around $80 \mathrm{pA}$ and for supply $V_{D D}$ is around $10 \mathrm{pA}$, so the typical total quiescent power consumption of this level-up shifter is around $0.5 \mathrm{nW}$. Considering the process and temperature variations by using Monte-Carlo simulations, the maximum quiescent power consumption can go up to $7.3 \mathrm{nW}$ at $150{ }^{\circ} \mathrm{C}$. However, this high temperature will unlikely happen in most implementations except for specific high-temperature purposes. Besides the static power loss, the total power consumption of a shifter should also include dynamic power loss, which depends on input signal frequency and gate capacitance of switches being driven. The total power consumed by all the level shifters employed in the system will be listed in a power consumption breakdown table in Section V.

In order to provide gate overdriving voltages $V_{D D A}$ and $V_{s u b}$, switched capacitor (SC) DC-DC converters are employed. Fig. 11 shows the circuit diagrams of the two DCDC converters. These two DC-DC converters perform voltage conversions with ratios $\frac{3}{1}$ and $-\frac{1}{2}$ respectively. Due to the limited chip design area reserved for this circuit, the capacitors used in the converters are off-chip SMD capacitors with $C_{1}=$ $C_{2}=1 \mathrm{nF}$. Hence, there are totally 7 off-chip $1 \mathrm{nF}$ capacitors employed for the DC-DC converters in this implementation. However, simulations show that the total quiescent current flowing through $V_{D D A}$ and $V_{s u b}$ for the whole circuit is $0.3 \mathrm{nA}$ and the total average dynamic current is $2 \mathrm{nA}$, which make capacitors of $C_{1}=C_{2}=50 \mathrm{pF}$ sufficient to provide the required driving ability. Capacitors with these values can be readily designed on-chip to make the proposed interface circuit fully integrated.

The voltage converters are driven by two non-overlapping complementary clock signals, $\phi_{1}$ and $\phi_{2}$, which are generated from a single clock signal by cross-coupling the clock and its inverted version with two NOR gates and two weak inverters. Before $\phi_{1}$ and $\phi_{2}$ can be used to drive the converters, their levels need to be shifted through level shifters. In this implementation, the clock signal is generated by an internal on-chip ring oscillator, as shown in Fig. 12. The ring oscillator generates a raw clock signal at around $16 \mathrm{kHz}$; this clock is then divided by 16 to drive the DC-DC converters and further divided by 16 (to $62 \mathrm{~Hz}$ ) to drive the power management block. In order to supply the gate-overdriving voltage levels to make sure the parallel and series configurations are firmly held, the ring oscillator and SC converters are kept powered ON. Simulations show that the ring oscillator consumes an average power of $260 \mathrm{nW}$ and the voltage tripler and voltage half-inverter (in Fig. 11) consume $9 \mathrm{nW}$ and $4 \mathrm{nW}$ respectively with open outputs. Besides employing SC DC-DC converters, there are many other techniques to provide the switch gate-overdriving voltage levels, such as selecting the highest available voltage in the circuit nodes using a higher supply (HS) circuit for $V_{D D A}$ and using a negative voltage converter (NVC) for $V_{s u b}$, which are presented in [22]. As ring oscillators are normally power hungry and the low frequency ring oscillator employed in this paper consumes $260 \mathrm{nW}$ power (SC converters consumes $13 \mathrm{nW}$ extra power), using HS and NVC circuits can decrease this power consumption to $96 \mathrm{nW}$ (calculated according to [22]). However, the circuit in this paper requires a clock signal to drive the power management block presented in section IV-C in order to periodically put the system in sleep mode, 


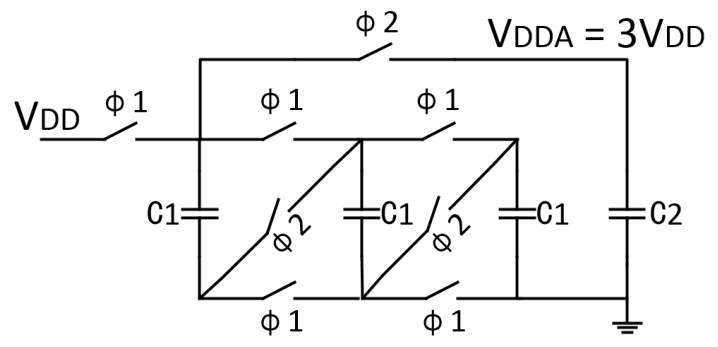

(a)

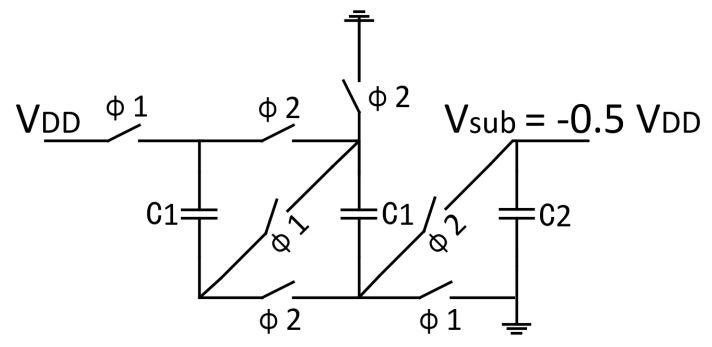

(b)

Fig. 11: Switched-capacitor (SC) converters used to generate gate overdriving voltage levels: (a) $V_{D D} \rightarrow 3 V_{D D}$, (b) $V_{D D} \rightarrow$ $-0.5 V_{D D}$

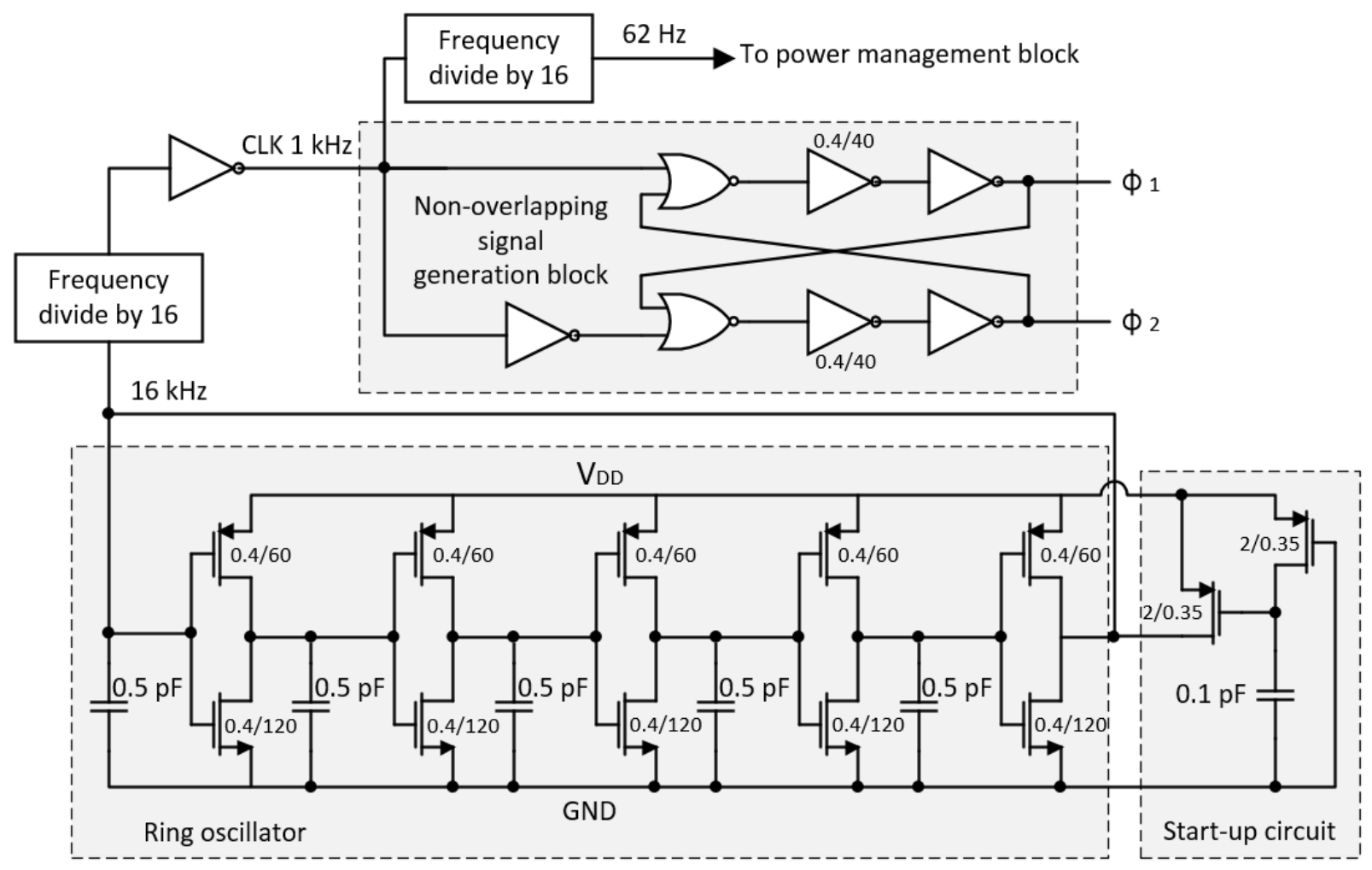

Fig. 12: Nano-power ring oscillator to provide digital clock for the SC converters and power management block, including the start-up circuitry and the non-overlapping signal generation block

and it cannot be guaranteed that the future load electronics can provide a such clock signal. As designing a ring oscillator is necessary in this implementation, SC DC-DC converters only consume $13 \mathrm{nW}$ extra power while the HS and NVC circuits would consume more.

\section{B. Calibration block}

In the calibration state, the two harvesters are forced to be connected in parallel, so $P 1$ and $P 2$ are connected to $P 12 ; N 1$ and $N 2$ are connected to $N$. For calibrating, $P 12$ is disconnected form $P$, so the PTs are in an open-circuit. As $N 1$ is still connected to $N$, the voltage at node $N 1$ equals to $-V_{D}$ due to the diode voltage drop between the ground reference and node $N 1$. Therefore, the peak-to-peak open-loop voltage between nodes $P 12$ and $N$ is now $V_{p p(o p e n)}=2\left(V_{P 12}+V_{D}\right)$ (as $V_{p p(o p e n)}=\left(V_{P 12}-V_{N}\right)_{\max }-\left(V_{N}-V_{P 12}\right)_{\max }=$ $\left.2\left(V_{P 12}-V_{N}\right)_{\max }=2\left(V_{P 12}+V_{D}\right)\right)$. Replacing the term in (17), it becomes:

$$
\begin{aligned}
V_{p p(\text { open })} & >3\left(V_{S}+2 V_{D}\right) \\
2\left(V_{P 12}+V_{D}\right) & >3\left(V_{S}+2 V_{D}\right) \\
2 V_{P 12} & >3 V_{S}+4 V_{D} \\
2\left(V_{P 12}-2 V_{D}\right) & >3 V_{S} \\
\frac{1}{5}\left(V_{P 12}-2 V_{D}\right) & >\frac{3}{10} V_{S}
\end{aligned}
$$

Fractions on both sides of the inequality are to make sure that the values on the two sides are in the operational range of the comparator. Fig. 13 shows the circuit diagram of calibration block to perform the comparison of (19). The 


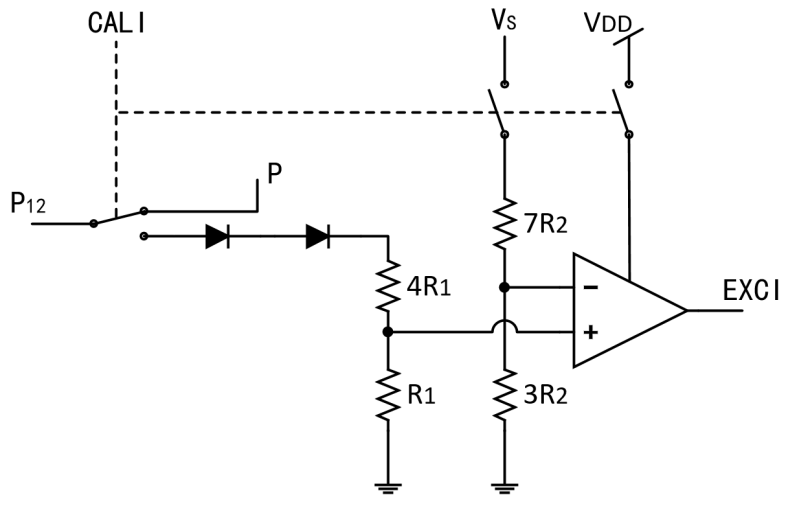

Fig. 13: Circuit diagram of the calibration block

two diodes used in the circuitry are the same as the ones used in the full-bridge rectifier in order to make sure they have same $V_{D}$. From this circuitry, the non-inverting input of the comparator is $\frac{1}{5}\left(V_{P 1}-2 V_{D}\right)$ and the inverting input is $\frac{3}{10} V_{S}$. The unit resistances $R_{1}$ and $R_{2}$ in the circuit are set to $0.6 \mathrm{M} \Omega$ and $0.5 \mathrm{M} \Omega$ respectively, hence the total resistances for each of the two resistive paths are $3 \mathrm{M} \Omega$ and $5 \mathrm{M} \Omega$. The resistors are on-chip implemented. The current on these two branches depends on the voltage at node $P_{12}$ and the voltage $V_{S}$. During the calibration mode while $P_{12}$ is disconnected from the full-bridge rectifier, the voltage on the $R_{1}$ branch approximately equals to the open-circuit voltage of the PT, which can have an amplitude varying from $0 \mathrm{~V}$ to $12 \mathrm{~V}$. Choosing the middle value $6 \mathrm{~V}$ for estimation, the power loss due to this path is $3 \mu \mathrm{W}$ ( $V_{P 12}$ is a sine signal between $0 \mathrm{~V}$ and $6 \mathrm{~V}$ ). In terms of the $R_{2}$ branch, the $V_{S}$ usually varies from $2 \mathrm{~V}$ to $6 \mathrm{~V}$; hence the average power loss due to this path is $3.2 \mu \mathrm{W}$ (taking $V_{S}=4 \mathrm{~V}$ ). As the two branches are cut from $P_{12}$ and $V_{S}$ in sleep mode, which takes a very majority of time, the total average power loss on these two branches equals $6.2 \mu \mathrm{W} \times d_{\text {cali }}$, where $d_{\text {cali }}$ represents the duty ratio of the calibration mode. The base power loss $6.2 \mu \mathrm{W}$ for these two resistive branches can be further reduced by increasing the resistances or using off-chip resistors to provide much higher resistances. Although larger value resistors are able to reduce the base power loss to less than $1 \mu \mathrm{W}$, they can take up additional area, either on the chip or on the test board with off-chip resistors. The resistance $R_{2}$ can be increased to a much higher value as the variation of $V_{S}$ is slow. However, the value of $R_{1}$ should be below a reasonable limit because the frequency of $V_{P 12}$ signal can be quite high and the input transistors of the comparator have large sizes (500/0.5). Hence, high $R_{1}$ along with the large input capacitance of the comparator form a passive RC low-pass filter which filters out high frequency $V_{P 12}$ signal.

The output signal of this block, $E X C I$, indicates that the environmental excitation is high enough to satisfy the condition in (19). For generating the EXCI signal, a continuoustime comparator is employed [23], which is shown in figure 14. A trade-off between the power loss and the performance determines the biasing current. With a $25 \mathrm{nA}$ biasing current, the settling time of the comparator is around $40 \mu \mathrm{s}$, which

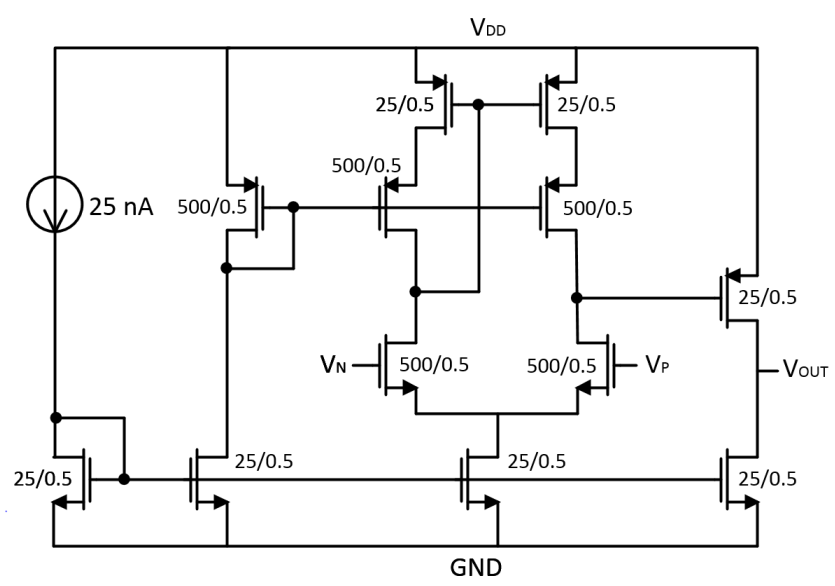

Fig. 14: Continuous time comparator to evaluate the input excitation level

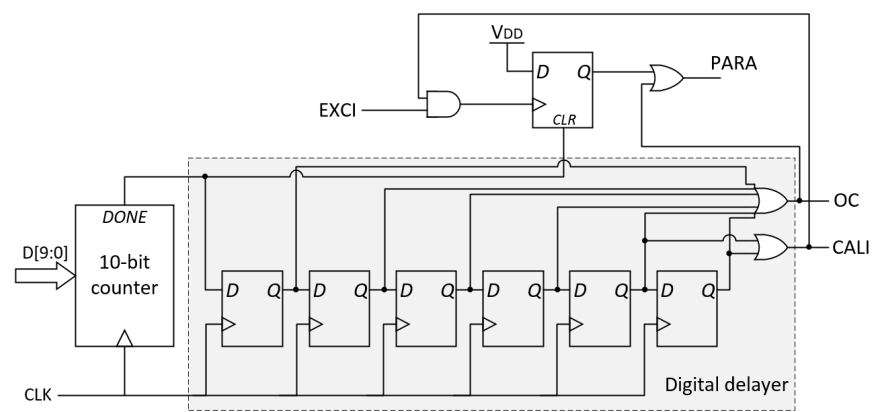

Fig. 15: Power management and parallel-series determining circuitry

is acceptable for most of PTs as it is much shorter than the periods of PTs and the static power loss is decreased to $150 \mathrm{nW}$. In addition, the comparator is powered OFF in the sleep mode to further decrease power loss.

\section{Power management block}

Fig. 15 shows a power management circuitry employed to power OFF some parts of the system for a certain time while they are not in use and to generate digital control signals. As shown in the figure, the power management circuit utilizes a 10-bit digital counter for determining the duration of sleep mode. The clock signal of this 10-bit counter is of around $62 \mathrm{~Hz}$. The 10-bit counting number D[9:0] of the counter is set externally and the system goes into "calibration mode" once it counts to the preset value D[9:0]. For instance, if $\mathrm{D}[9: 0]=$ 255 , the counter will be reset after 255 cycles of $C L K$, which is approximately 4 seconds. The maximum value can be set to 1023 , or 16 seconds. Once the counter finishes counting, a pulse $D O N E$ is generated and the counter is synchronously reset to restart counting from 0 . In order to make the power management block working as expected to let system go into the two different modes alternatively for reasonable durations, the four LSBs D[3:0] are internally set to 4'b1111 and cannot be configured.

After the counter, a digital delayer using six simple Dflip-flops is employed. When the counter finishes counting, 


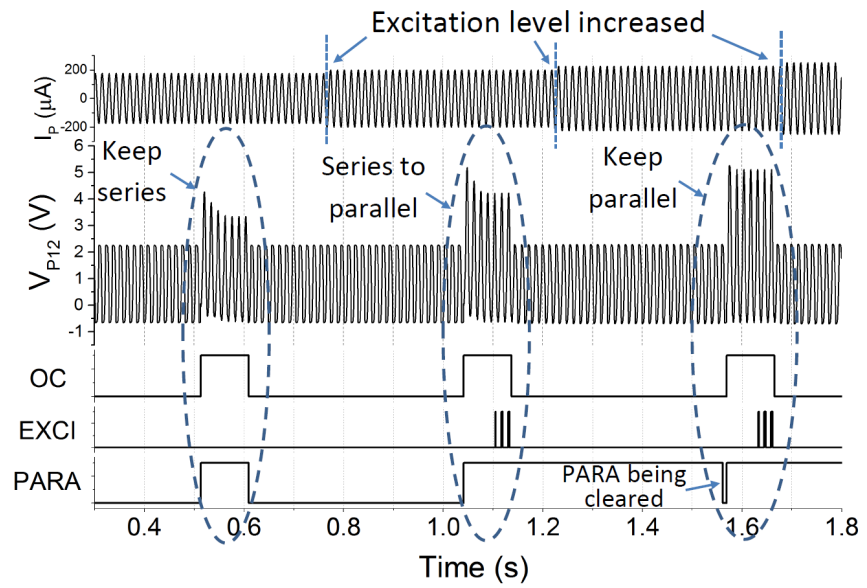

Fig. 16: Simulation results of the proposed circuit

a pulse of $D O N E$ generates a pulse of $O C$ (short for 'opencircuit') lasting for 6 clock periods and a pulse of $C A L I$ (short for 'calibration') lasting for 2 clock periods. If the input of the counter is set to the maximum value $\mathrm{D}[9: 0]=$ 1023 , the calibration mode only takes $6 / 1024 \approx 0.6 \%$ of the total time, which means the average power consumption and current leakage associated to the calibration mode are largely reduced to $0.6 \%$. The pulse $O C$ is used to set the prerequisite conditions for performing the algorithm in the calibration block: forcing the two PTs to be connected in parallel and in an open-circuit. During the pulse of $O C$, the calibration block is powered ON but its output EXCI is disabled until the pulse $C A L I$ is present (the last two period of the pulse $O C$ ). Because suddenly putting the PTs in open-circuit from a closed circuit may cause issues on the reference voltage, which can make the voltage at the node $P_{12}$ going to an unexpected voltage level and the voltage at node $N$ does not equal to $-V_{D}$. This is because before an $O C$ pulse, node $P_{12}$ is connected to one electrode of the storage capacitor $C_{S}$ through a diode and node $N$ (the other electrode of PTs) is connected to the other electrode of $C_{S}$ through a another diode (refer to Fig. 8). In this case, the two diodes connect $P_{12}, N$ and $C_{S}$ and form a closed loop. As there is current flowing in this loop when energy is transferred to $C_{S}, N$ should be equal to $-V_{D}$ (assuming $N$ is the lower potential node). Once $P_{12}$ is disconnected from the diode connected to $C_{S}$, there is no closed loop between $P_{12}$ and $N$ nodes. Although $N$ is connected to the ground through a diode, there is no current flowing through the diode to ensure that the potential at $N$ is $-V_{D}$. In this case, the voltage potential at $N$ is not stable and it equals to the ground $(0 \mathrm{~V})$ at the instant of $O C$ goes high. This introduces an $V_{D}$ offset to the voltage at $P_{12}$ because the inequality derived in (19) requires the voltage at $N$ is $-V_{D}$. In order to make $V_{N}$ be able to attain $-V_{D}$ while $V_{P(12)}$ goes high, some time is needed after $O C$ goes high to let the diode between $N$ and the ground "slowly" set $V_{N}=-V_{D}$. In this implementation, four periods of $C L K$ is given. In the following two periods of $C L K, C A L I$ pulse is generated which enables $E X C I$.

Fig. 16 shows the simulated waveforms of the proposed circuit. The signal $I_{P}$ at the top represents the excitation amplitude, in unit of $\mu \mathrm{A}$, which is increased gradually. The second signal $V_{P 12}$ is the voltage at the node $P 12$ shown in Fig. 13. From the figure, the calibration mode is entered three times, where $O C$ is high, in this simulation. When $O C$ is high, the PTs are in open circuit and $V_{P 12}$ exceeds the limit $V_{S}+V_{D}$. It can be seen that $V_{P 12}$ needs a little time to stabilize before the signal $E X C I$ is enabled and can be generated at the end of the calibration state. During the first calibration mode, although the signal $P A R A$ is forced to high to evaluate the excitation level, it goes low again after the calibration mode as the EXCI pulse is not generated due to low excitation amplitude. After this calibration mode, $I_{P}$ is increased. During the second calibration mode, it can be seen that three pulses of EXCI is generated because the circuit chooses a parallel connection according to the excitation input. It is worth mentioning that the EXCI pulses are generated according to the amplitude of $V_{P 12}$, which has a frequency of $82 \mathrm{~Hz}$. As mentioned before, the EXCI signal is only enabled for two $C L K$ cycles and the frequency of $C L K$ is $62 \mathrm{~Hz}$. This explains why three EXCI pulses are generated in two $C L K$ cycles. If the excitation frequency goes higher (or lower), there will be more (or less) EXCI pulses generated in two $C L K$ cycles if the amplitude is high enough. If the excitation frequency is less than $31 \mathrm{~Hz}$ (half frequency of $C L K$ ) such that the period of the excitation is longer than two $C L K$ cycles, an excitation peak cannot always be observed in the two $C L K$ cycles. Hence, EXCI pulses cannot always be generated in this case. This may occasionally result in an unexpected series connection while the parallel connection is preferred under high excitation levels. Therefore, the proposed system requires the excitation frequency higher than $31 \mathrm{~Hz}$ to ensure correct connection switching. Before the third $O C$ pulse, the excitation level is further increased and the PTs are expected to be connected in parallel. Right before the third $O C$ pulse, $P A R A$ goes low for one clock cycle and it goes back to high level. This is because the top single D-flip-flop in Fig. 15 is reset first before each calibration mode, which allows $P A R A$ to be cleared to low level before it is forced to go high by $O C$ signal.

The single D-flip-flop in this block is used to provide a decision on the connection type based on the signal EXCI, which is generated in the calibration block in Fig. 13. While the counter finishes counting, the signal DONE resets the flip-flop to a low level regardless the previous connection type (parallel or series). The $C A L I$ pulse is used to enable the $E X C I$ signal. If one or more $E X C I$ pulses are present in the calibration mode during the pulse of $C A L I$, the output of the flip-flop goes high and keeps the two PTs connected in parallel after the calibration state. If the excitation is too low to generate a pulse of $E X C I, P A R A$ signal will go back to low level after the calibration mode ends. An external one-time reset is performed on all of the flip-flops once the circuit is implemented and powered ON. During the simulation shown in Fig. 16, the input of the counter is set to $D[9: 0]=32$. This value is very small and is very impractical because the calibration mode takes a large percentage of the time (duty ratio is around $6 / 32 \approx 18.7 \%$ ). During this mode, some extra 


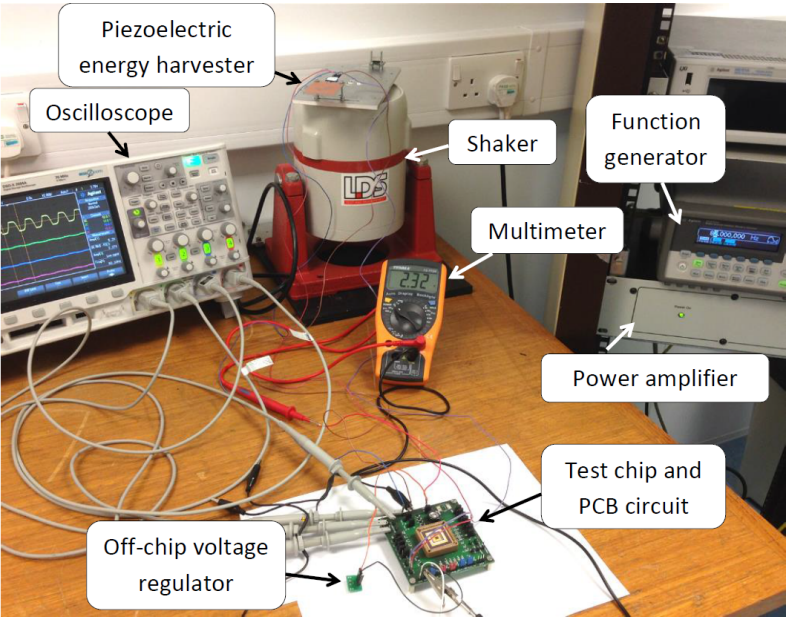

Fig. 17: Experimental setup

energy is consumed and no energy can be transferred from the PT to the storage capacitor due to the open circuit of the PT. However, this small value chosen here is to clearly show the working principle of the proposed interface circuit and to decrease the simulation time due to the slow simulation speed. As discussed above, if the maximum value $D[9: 0]=1023$ is chosen for the counter, the duty ratio of the calibration mode is only $0.6 \%$.

As discussed above, a smaller calibration duty ratio results in lower power consumption but the system also reacts slowly to variations in environmental excitation. Hence, there exists a trade-off between power consumption and circuit response time. The general principle is to keep the calibration duty ratio as small as possible while the circuit is able to react to the environmental excitation amplitude variation. As the four LSBs of the input signal D[3:0] for the counter are internally set to 4'b1111, the shortest sleep time is around $0.25 \mathrm{~s}$ with a $37 \%$ calibration duty ratio. Hence, the proposed system cannot react to significant variation in excitation level faster than this value. However, the shortest sleep time preset by the circuit is impractical due to the large calibration duty ratio. Therefore, the proposed system is not suitable in environments with uncertain base vibration without target periods of time when the excitation level is high.

\section{MEASUREMENT RESUlts AND DISCUSSIONS}

The proposed connection auto-switching interface circuit was experimentally evaluated (see Fig. 17) using a commercially available bimorph cantilevered piezoelectric harvester with dimension $47 \mathrm{~mm} \times 36 \mathrm{~mm}$ (Mide Technology Corporation $\mathrm{V} 20 \mathrm{~W}$ ). A shaker (LDS V406 M4-CE) was excited at the natural frequency of the cantilever at $82 \mathrm{~Hz}$ and driven by a sine wave from a function generator (Agilent Technologies $33250 \mathrm{~A} 80 \mathrm{MHz}$ waveform generator) amplified by a power amplifier (LDS PA100E Power Amplifier). The test chip was powered by an external power supply at $1.5 \mathrm{~V}$ (can go up to $1.8 \mathrm{~V}$ for higher $V_{S}$ ) and an off-chip voltage regulator (ON Semiconductor NCP4681DSQ15T1G) with ultra-low ground leakage current $\left(I_{G N D} \approx 1.5 \mu \mathrm{A}\right)$ is also available to allow

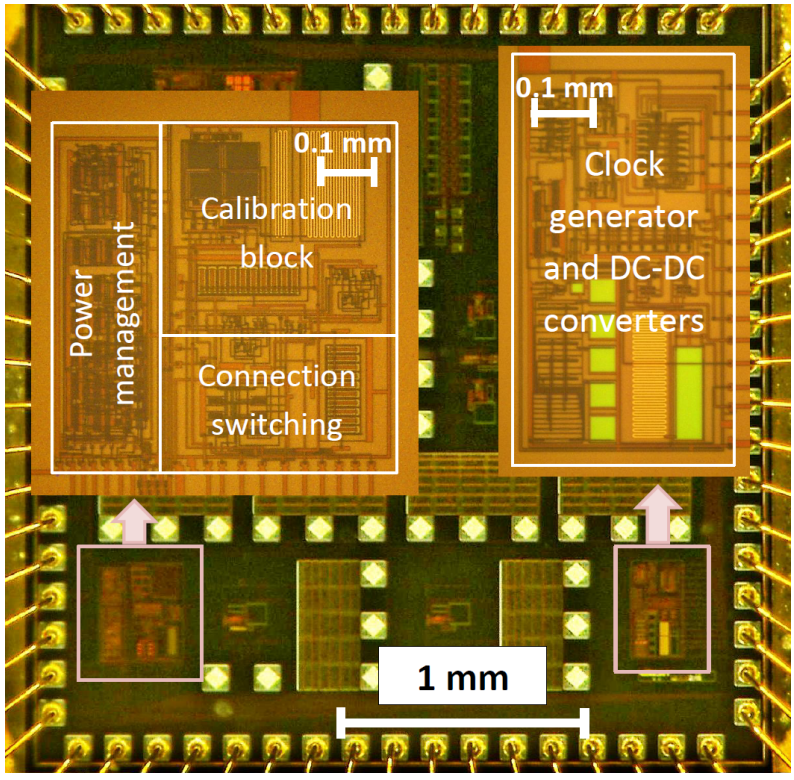

Fig. 18: Micrograph of the test chip fabricated in a $0.35 \mu \mathrm{m}$ CMOS foundry process. The overall die size is $2.8 \mathrm{~mm} \times$ $3.2 \mathrm{~mm}$. The active area for the proposed circuit is around $0.5 \mathrm{~mm}^{2}$ excluding the pads and the remaining die area is occupied by circuits of other projects

TABLE I: Breakdown of the chip power loss and other power loss sources with simulated and measured results

\begin{tabular}{ccc}
\hline \hline Loss mechanism & Power loss & Percentage \\
\hline Ring oscillator & $260 \mathrm{nW}$ & $57.5 \%$ \\
DC converters & $13 \mathrm{nW}$ & $2.9 \%$ \\
Level shifters & $25.7 \mathrm{nW}$ & $5.7 \%$ \\
Power management & $0.6 \mathrm{nW}$ & $0.1 \%$ \\
Switching block & $0.3 \mathrm{nW}$ & $0.1 \%$ \\
Calibration block $*$ & $152.4 \mathrm{nW}$ & $33.7 \%$ \\
Total (circuit) & $452 \mathrm{nW}$ & $100 \%$ \\
Measured circuit loss & $\sim 0.5 \mu \mathrm{W}$ & \\
$C_{S}$ leakage & $\sim 0.24 \mu \mathrm{W}$ \\
\hline (* depends on the calibration duty ratio)
\end{tabular}

for the system to be self-powered. The components on the PCB board include a storage super capacitor (AVX BestCap BZ05CA103ZSB, measured capacitance $C_{S} \approx 5.2 \mathrm{mF}$ ), a few $1 \mathrm{nF}$ SMD capacitors for SC DC-DC converters, external digital inputs and pins for observing some key signals.

The proposed chip was implemented in a $0.35 \mu \mathrm{m}$ CMOS process. Fig. 18 shows the die photo of the test chip. The active area of the proposed connection auto-switching circuit together with the DC-DC converters and the clock generator is around $0.5 \mathrm{~mm}^{2}$. The micrograph of the chip identifies the area occupied by the clock generator, DC-DC converters, power management block, calibration block and connection switching block.

Table I lists the simulated power loss due to different parts of the energy harvesting system. The values for the individual circuit blocks are simulated results. In terms of the power loss due to the calibration block, the duty ratio of 


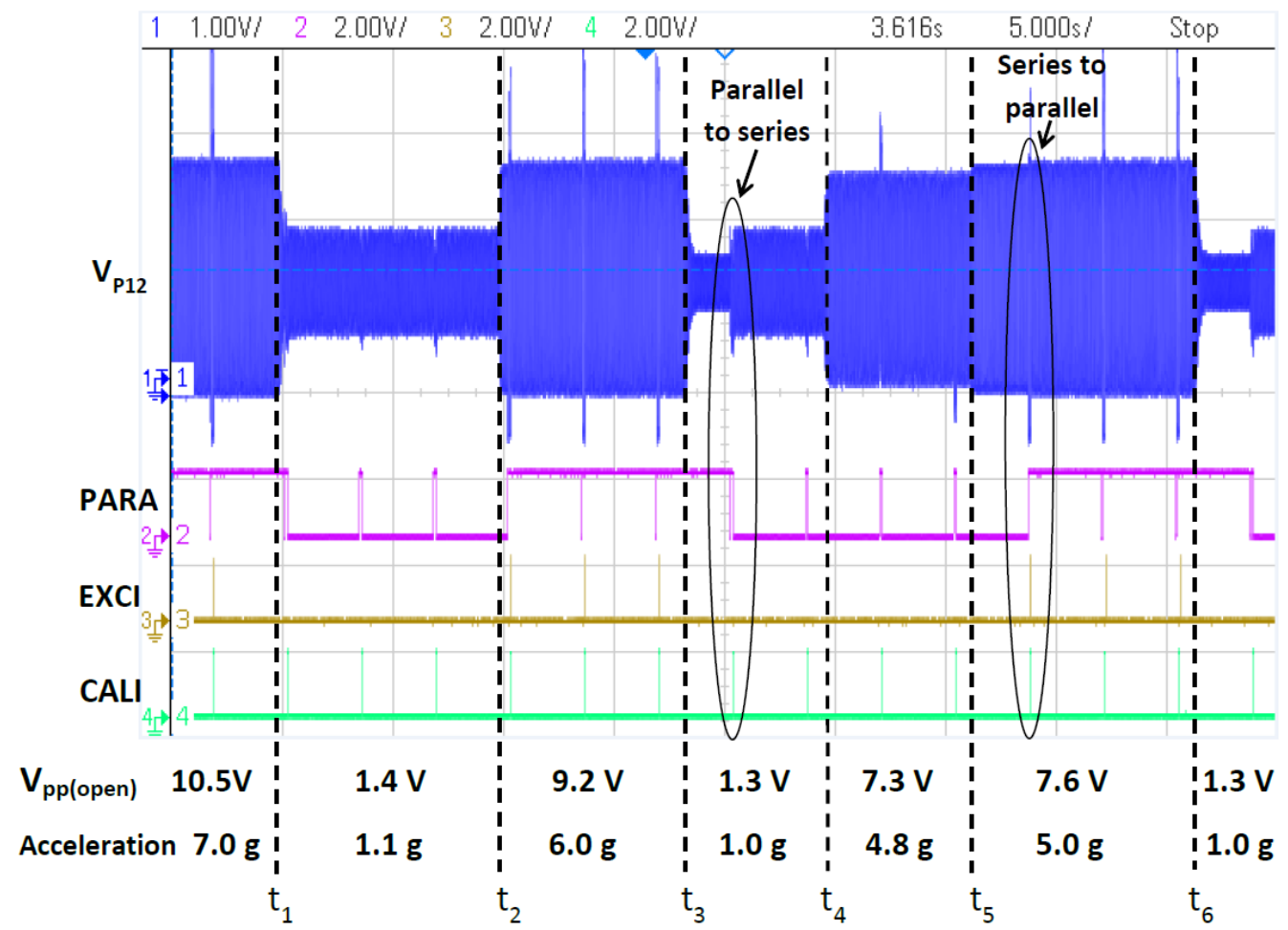

Fig. 19: Measured waveforms of signal $V_{P 12}, P A R A, E X C I$ and $C A L I$ in a period of $50 \mathrm{~s}$

calibration mode during the measurement is chosen at $2.4 \%$, corresponding to $\mathrm{D}[9: 0]=256$ for the digital counter in the power management block. With a $62 \mathrm{~Hz}$ clock, the calibration mode is expected to be entered every $4 \mathrm{~s}$ and this can be observed from Fig. 19. Hence the effective power loss due to this block is $6.35 \mu \mathrm{W} \times 2.4 \%=152.4 \mathrm{nW}$. The simulated total power consumption of the interface circuit with a $2.4 \%$ calibration mode duty ration is $452 \mathrm{nW}$, which is smaller than the measured value $500 \mathrm{nW}$. This is possibly due to the excitation level and the voltage across the storage capacitor $C_{S}$ during the measurements are relatively high, which increase the power loss due to the resistive branches in the calibration block as shown in Fig. 13. The storage capacitor $C_{S}$ is an off-chip super capacitor of $5.2 \mathrm{mF}$ and the power loss due to its internal leakage has been experimentally evaluated. The measurement was started by charging $C_{S}$ to $4.21 \mathrm{~V}$. After 1 day 19 hours and 7 minutes of leaving it disconnected from any electronic devices, the voltage decreased to $1.86 \mathrm{~V}$ and the power loss is calculated by dividing the energy loss in the capacitor over the time. As the leakage current of $C_{S}$ depends on the voltage across it, the measured power loss $[0.24 \mu \mathrm{W}]$ should be regarded as an average value for $V_{S}$ between $1.86 \mathrm{~V}$ and $4.21 \mathrm{~V}$.

Fig. 19 shows measured waveforms from an oscilloscope of four signals: $V_{P 12}, P A R A, E X C I$ and $C A L I$ (from top to bottom). The signals were measured in a period of $50 \mathrm{~s}$ by changing the input excitation amplitude. The signal $V_{P 12}$ is the voltage at the node $P_{12}$; the signal PARA indicates the connection type that is being used; the signal EXCI is the output signal from the calibration block indicating that the condition in (17) is satisfied and the signal CALI is the output signal from the power management block indicating that the system is in "calibration mode". From the CALI signal, it can be seen that the "calibration mode" was entered periodically for every $4 \mathrm{~s}$ (approximately). According to the section IV-C, the "calibration mode" starts when the digital counter finishes counting. The first cycle after the counting ends, a signal $D O N E$ is generated to reset a flip-flop to have a series connection (refer to Fig. 15). This explains why the signal $P A R A$ goes low for a very short time (actually for one clock cycle) when "calibration mode" starts with a high level $P A R A$. After the $P A R A$ is reset to low level, the signal delayer in the power management block forces $P A R A$ to high level for a few cycles to evaluate the excitation. This forced high PARA pulses can also be seen from the Fig. 19 corresponding to pulses $C A L I$. The signal EXCI indicates the result after evaluating the excitation amplitude according to the algorithm in (19). If a pulse of EXCI is present for a "calibration mode", the signal PARA keeps high after the mode ends; otherwise, $P A R A$ goes low because the excitation is too low to generated a $E X C I$ pulse.

During the $50 \mathrm{~s}$ measurement, the excitation amplitude was changed 6 times, which are marked as $t_{1}, t_{2}, t_{3}, t_{4}, t_{5}$ and $t_{6}$ in the figure. The excitation amplitudes for all the time intervals are shown at the bottom of the figure as the peak-to-peak open-circuit voltage of the PT $V_{p p(o p e n)}$ and the corresponding acceleration level in unit of the gravity. As $t_{1}$ and $t_{2}$ are slightly before calibration states start, the effect of different connection types on the signal $V_{P 12}$ is not observable. Hence, explanations on the figure will be based on the period after $t_{2}$. From the figure, several $C A L I$ pulses can be found between $t_{2}$ and $t_{3}$, where pulses of $E X C I$ are 


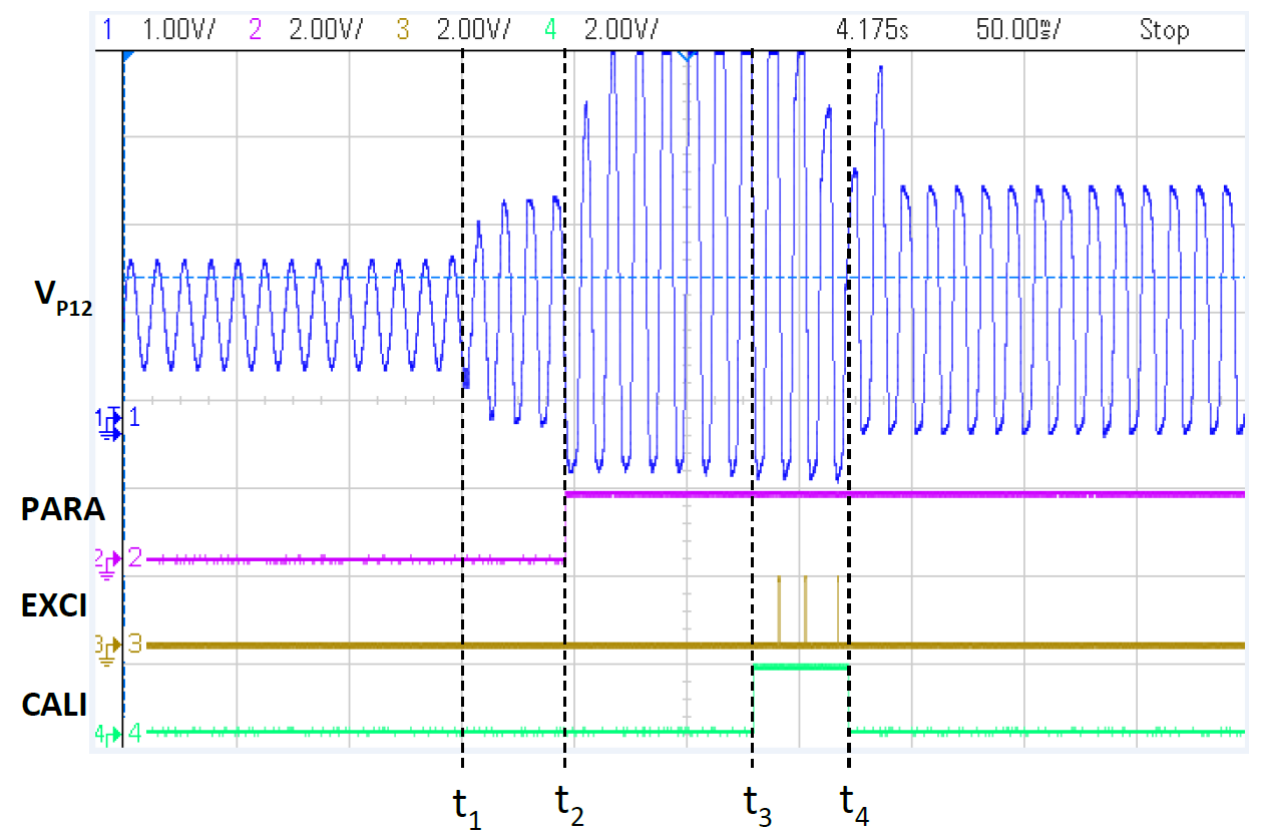

Fig. 20: Measured waveforms in $0.5 \mathrm{~s}$ while connection is being changed from series to parallel

generated. This means the excitation level is high to satisfy the condition in (19). As a result, PARA keeps at high level after each calibration mode. During the calibration modes, it can be seen that spikes are present in the signal $V_{P 12}$. This is because the PTs are disconnected from the full-bridge rectifier during the calibration mode (open-circuit), hence $V_{P 12}$ is not limited below $V_{S}+V_{D}$ and it can go higher. Similar spikes can also be observed for some of the other calibration states but spikes are not present for low excitations when $V_{P 12}$ cannot attain $V_{S}+V_{D}$. The excitation amplitude is then significantly decreased at time $t_{3}$ and a sudden drop in $V_{P 12}$ can be observed. During the calibration state after $t_{3}$ (marked in the left ellipse), no $E X C I$ pulse is present, which results in a series connection. Once the PTs are connected in series, the amplitude of signal $V_{P 12}$ can be observed to be doubled because series connection doubles the voltage across the PTs.

The excitation is then increased at time $t_{4}$ where a sudden amplitude increase of $V_{P 12}$ can be observed. However, the $E X C I$ signal still keeps low for the following two calibration states because the excitation level is not high enough. The excitation is further increased at $t_{5}$. The following calibration state confirms that the condition in (19) is satisfied and a pulse $E X C I$ is generated. As a result, the $P A R A$ goes high (marked in the second ellipse).

Fig. 20 shows the waveforms of the four signals in a short period of time while the connection is being changed from series to parallel. At time $t_{1}$, the excitation is increased and the resulting $V_{P 12}$ can be observed from the figure. From time $t_{2}$ to $t_{4}$, the connection is forced to be parallel and PARA goes high. During this time, the PTs are in an open-circuit hence $V_{P 12}$ can go very high. Between $t_{2}$ and $t_{3}$, the excitation evaluation is not enabled because a little time is needed to let $V_{P 12}$ become stable (detailed explanations are in section IV-C). Between $t_{3}$ and $t_{4}$, the signal $C A L I$ goes high to

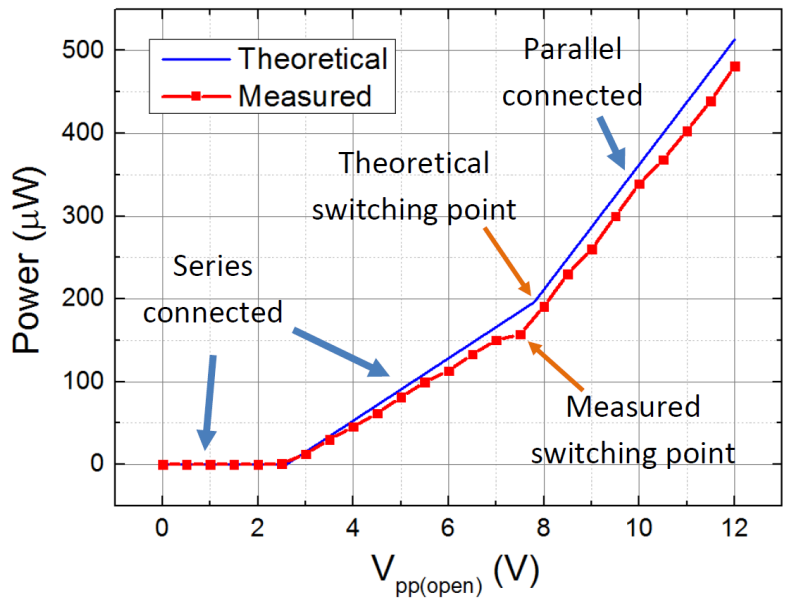

Fig. 21: Output power comparison between theoretical and measured results as a function of excitation level with fixed $V_{S}=2 \mathrm{~V}$ (diode voltage drop $V_{D}=0.3 \mathrm{~V}$ )

enable the EXCI signal; therefore, three $E X C I$ pulses are generated due to satisfying the condition in (19). These pulses indicate that the connection will be parallel after the calibration state; hence, PARA keeps high after $t_{4}$.

In order to measure the output electrical power transferred to the storage capacitor $C_{S}$ at a given $V_{p p(o p e n)}$ and $V_{S}$, the voltage increase in $C_{S}$ in a short period of time is measured to calculate the energy increase in this time. The formula of calculating the output power is: $P=C_{S}\left(V_{S(\text { end })}^{2}-V_{S(\text { start })}^{2}\right) / 2 T$, where $V_{S(\text { start })}$ is the starting voltage of $V_{S}, V_{S(\text { end })}$ is the ending voltage of $V_{S}$ and $T$ is the time used to charge $C_{S}$ from $V_{S(\text { start })}$ to $V_{S(e n d)}$. As $V_{S}$ is increasing during measurement and the output power should be obtained at some fixed $V_{S}$ values, the $V_{S \text { (start) }}$ and $V_{S(e n d)}$ are chosen to be close to 


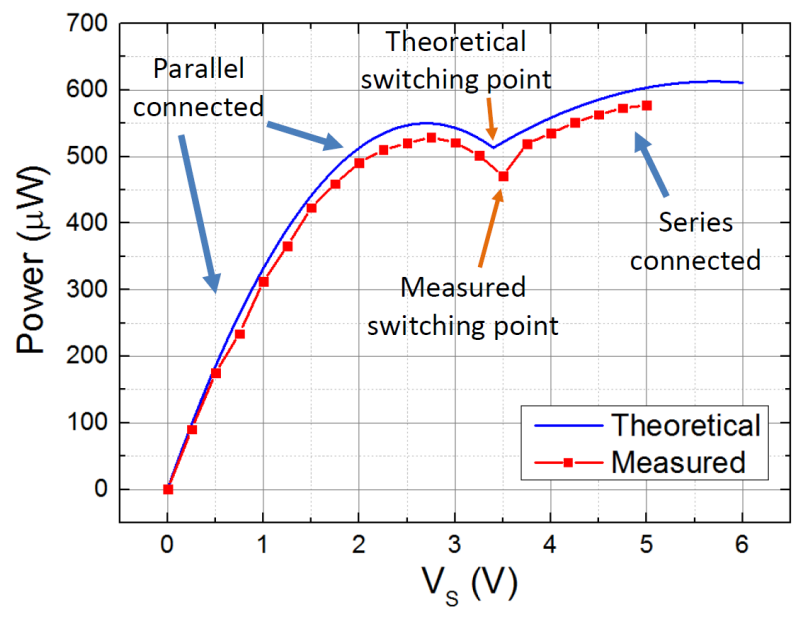

(a)

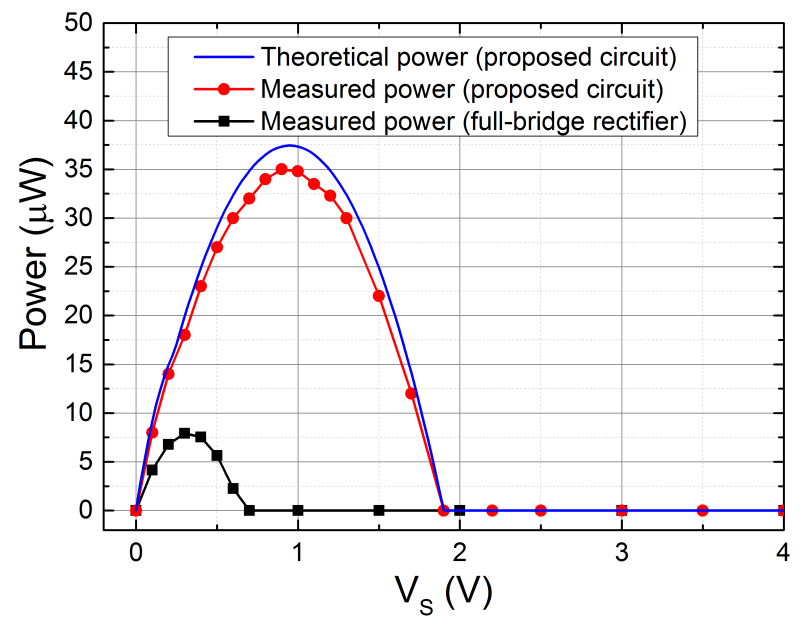

(b)

Fig. 22: Theoretical and measured output power in function $V_{S}$ using proposed circuit and full-bridge rectifier under different excitation levels. (a) With a fixed excitation level of $V_{p p(o p e n)}=12 \mathrm{~V}$ (equivalent to acceleration level of $8.0 \mathrm{~g}$ ); (b) $V_{p p(o p e n)}=$ $2.5 \mathrm{~V}$ (equivalent to $1.8 \mathrm{~g}$ ). Diode voltage drop $V_{D}=0.3 \mathrm{~V}$

make the results accurate. While measuring the output power at $V_{S}=2 \mathrm{~V}$, for instance, $V_{S(\text { start })}$ and $V_{S(\text { end })}$ are chosen at $1.9 \mathrm{~V}$ and $2.1 \mathrm{~V}$ with a fixed excitation level $V_{p p(o p e n)}$. The time consumed to charge $C_{S}$ from $1.9 \mathrm{~V}$ to $2.1 \mathrm{~V}$ is recorded by a stopwatch (of a smart phone) and the output power transferred to $C_{S}$ can therefore be calculated.

Fig. 21 shows the measured output power compared to theoretical results with fixed $V_{S}=2 \mathrm{~V}$ and changing excitation level, where the highest $V_{p p(o p e n)}(12 \mathrm{~V})$ corresponds to acceleration of $8.0 \mathrm{~g}$. As $V_{S}=2 \mathrm{~V}$ and $V_{D}=0.3 \mathrm{~V}$ are used in experiments, the expected threshold voltage for pure parallel model is $2\left(V_{S}+2 V_{D}\right)=5.2 \mathrm{~V}$ and the expected switching point for parallel and series connection is $3\left(V_{S}+2 V_{D}\right)=7.8 \mathrm{~V}$. Compared to the pure parallel model, the proposed circuit can let the rectifier start extracting energy from the PTs at a lower threshold voltage $2.6 \mathrm{~V}$. Compared to the pure series model, the circuit extracts more energy while excitation amplitudes are higher than the switching point such that $V_{p p(o p e n)}>7.8 \mathrm{~V}$. The measured results show that the switching point is shifted to near $7.5 \mathrm{~V}$ and this is due to nonideal diodes used in measurements. Non-ideal diodes allow forward leakage current flowing through while the forward voltage is lower than $V_{D}$; hence the effective $V_{D}$ is lower than $0.3 \mathrm{~V}$, which makes the switching point shifting leftwards on the graph.

Fig. 22 shows the measured results with the proposed circuit and a full-bridge rectifier at fixed excitation levels $\left(V_{p p(o p e n)}=\right.$ $12 \mathrm{~V}$ in the left figure and $V_{p p(o p e n)}=2.5 \mathrm{~V}$ in the right figure) with $V_{S}$ varying from $0 \mathrm{~V}$ to $5 \mathrm{~V}$. The results in Fig. 22a show that the switching point is measured at $V_{S}=3.5 \mathrm{~V}$, which is slightly higher than the theoretical value $3.3 \mathrm{~V}$. This is also due to the non-ideal diodes used in measurements. The switching point is set as $V_{p p(o p e n)}=3\left(V_{S}+2 V_{D}\right)$. While non-ideal diodes have lower $V_{D}$ values, $V_{S}$ goes higher to keep a constant $V_{p p(o p e n)}$. This explains the difference between theoretical and measured results. At a high excitation level
$\left(V_{p p(o p e n)}=12 \mathrm{~V}\right)$ in Fig. 22a, there exists a maximum power point for each of the two connection types. With the proposed interface circuit, the energy harvesting system is able to attain both of the two peak power points at $V_{S}=2.6 \mathrm{~V}$ and $V_{S}=5.5 \mathrm{~V}$, which enable a wide range of $V_{S}$ to obtain high output power. The same experiments were performed at a low excitation level $\left.\left(V_{p p(o p e n}\right)=2.5 \mathrm{~V}\right)$ in Fig. $22 \mathrm{~b}$. The results show that the output electrical power using the proposed circuit can attain a peak power of $34.9 \mu \mathrm{W}$, which is $4.5 \times$ higher than the power obtained from a simple full-bridge rectifier, which is $7.8 \mu \mathrm{W}$. This is due to the series connection chosen by the circuit because the series model outputs much higher power than the parallel counterpart at low excitation levels. In addition, it can be seen that the extra power consumption introduced by the interface circuit $0.5 \mu \mathrm{W}$ shown in Table I is far lower than the extra power extracted by this circuit compared to using a simple full-bridge rectifier.

Fig. 23 shows the measured electrical output power while $V_{p p(o p e n)}$ is varied from $0 \mathrm{~V}$ to $12 \mathrm{~V}$ with steps of $1 \mathrm{~V}$ and $V_{S}$ is varied from $0 \mathrm{~V}$ to $5 \mathrm{~V}$ with steps of $0.5 \mathrm{~V}$. There are $13 V_{p p(o p e n)}$ values and $11 V_{S}$ values chosen, hence totally 143 output power values measured. This figure illustrates the performance of the circuit in the full ranges of excitation level and $V_{S}$. The results shown in Fig. 21 and Fig. 22 are highlighted in the $V_{S}=2 \mathrm{~V}, V_{p p(o p e n)}=12 \mathrm{~V}$ and $V_{p p(o p e n)}=2.5 \mathrm{~V}$ planes. The middle dashed curve separates the parallel and series configurations according to different values of $V_{p p(o p e n)}$ and $V_{S}$.

Fig. 24 shows the measured power efficiency of the proposed interface circuit while an external power supply is used and it is self-powered with an off-chip voltage regulator. While the circuit is self-powered using an off-chip voltage regulator, the efficiency is reduced significantly. Although the leakage current of chosen voltage regulator is as low as $1.5 \mu \mathrm{A}$, the energy conversion efficiency is relatively low, which pulls down the overall efficiency. [24] presents an on-chip high-efficiency 


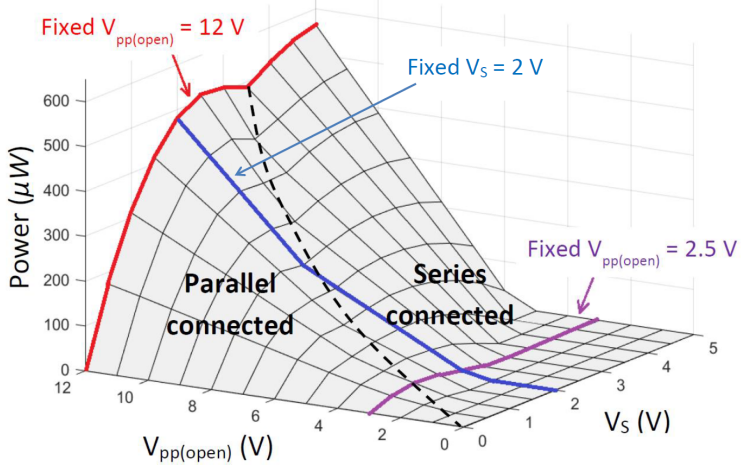

Fig. 23: 3-D surface plot of measured output power in function of $V_{p p(o p e n)}$ and $V_{S}$

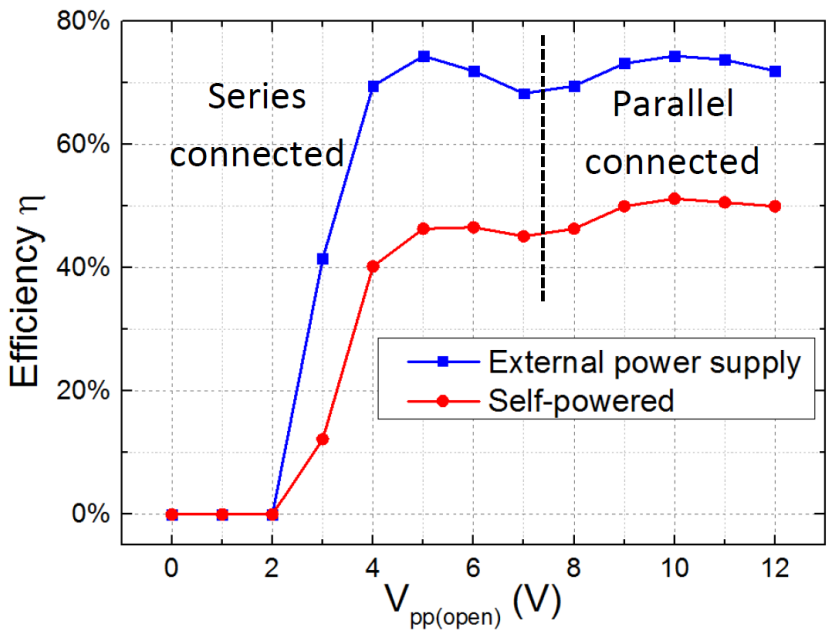

Fig. 24: Measured power efficiency of the proposed interface circuit while it is externally powered and self-powered $\left(V_{S}=\right.$ $2 \mathrm{~V}$ )

SC DC-DC converter with a nominal output voltage $1.5 \mathrm{~V}$ and efficiency up to $92 \%$, which can be a very good substitute of the off-chip voltage regulator to increase the overall efficiency. However, the voltage regulator employed here is just to allow for the possibility of the proposed circuit being self-powered for an energy autonomous module. The performance of the interface circuit itself should be the highlight of this paper. From the figure, it can be clearly found that there are two peak efficiency points because the circuit is trying to configure the PTs in a better way to output higher power. While $V_{p p(o p e n)}$ goes high from $0 \mathrm{~V}$, the power efficiency goes higher and attains its first peak near $V_{p p \text { (open })}=5 \mathrm{~V}$. When the excitation level keeps being increased, the efficiency decreases. When $V_{p p(o p e n)}$ goes higher than $7 \mathrm{~V}$, the interface circuit configures the connection of the two PTs from series to parallel in order to keep the high power efficiency; therefore, the circuit is able to attain a second peak efficiency point. The dashed line in the figure shows the different connections the circuit chooses and it can be found that the first peak is due to series and the second peak is due to parallel connection. Compared to many other interface circuit, the proposed circuit enables a high power efficiency in a wide range of excitation levels.
Table II compares the performance of the proposed circuit against some reported interface circuits for piezoelectric vibration energy harvesters. Apart from the circuit presented in this paper, all of other circuits require inductors to improve performance and some may require inductors in the range of millihenries. A fully-integrated design in this paper makes a significant contribution to reducing the overall volume of the system. Although the PT employed in this implementation is relatively big compared to a SMD inductor and an inductorless design does seem to reduce the overall system volume significantly; however, using an inductorless and fully-integrated interface circuit is a very practical consideration for volumelimited MEMS piezoelectric energy harvesters [25]. All the devices needed for the proposed power management circuit are a $2.8 \mathrm{~mm} \times 3.2 \mathrm{~mm}$ chip (wire-bonder can be used instead of a chip carrier and socket), a $5.2 \mathrm{mF}$ storage capacitor and a SC-70-5 case voltage regulator (in the case that the voltage regulator is not implemented on-chip); hence the volume is expected to be less then $0.5 \mathrm{~cm}^{3}$.

As discussed in section II-C and experimentally verified in this section, the proposed scheme lowers the required excitation level by $50 \%$ and always chooses the connection type outputting higher power. In real world implementations, the ambient vibration amplitude is likely to vary with time and the proposed circuit is able to detect the excitation level in order to achieve high power efficiency in a wide range of excitation amplitudes. Concerning the performance boost compared to a full-bridge rectifier, the voltage drop of diodes used in the listed publications (including this work) are different, making any fair comparison difficult to carry out. For example, the diodes used in [28] are with nearly zero voltage drop. If [28] employs the same diodes as this paper, the performance boost should have a higher value and may be even higher than the performance achieved by the circuit in this paper. However, the highlight of the proposed interface circuit is not to achieved a highest possible output power; it aims to moderately increase the performance compared to a full-bridge rectifier while addressing the three drawbacks of SSHI and SECE circuits. A sub-micro watt inductorless fully-integrated interface circuit design allows for a significant decrease in the volume in compact system designs. In addition, the proposed circuit presents a different architecture and it dynamically configures the connection of two PTs to achieve higher power efficiency over a wide range of excitation amplitudes. Furthermore, as it does not generate synchronized current pulses in the piezoelectric materials, the proposed circuit is less subject to the SSD effect even for highly coupled PTs. Therefore, the mechanical vibration of the PTs will be less affected or damped, which extends the range over which the rectifier operates efficiently.

\section{CONCLUSION}

An adaptive sub-micro watt design for a piezoelectric energy harvesting interface circuit is proposed in this paper. The proposed circuit can be used to automatically connect two piezoelectric transducers (with same frequencies, amplitudes and phases) in parallel or in series according to the environmental excitation level and the voltage across the storage 
TABLE II: Performance comparison with reported interface circuits

\begin{tabular}{ccccccccc}
\hline \hline Publication & Techniques & $\begin{array}{c}\text { Power } \\
\text { consumption }\end{array}$ & PT & $V_{p p \text { open })}$ & $C_{P}$ & Frequency & Inductor? & $\begin{array}{c}\text { Performance } \\
\text { boost to FB }\end{array}$ \\
\hline \hline JSSC2010 [16] & Bias-flip & $2 \mu \mathrm{W}$ & Mide V22B & $2.4 \mathrm{~V}$ & $18 \mathrm{nF}$ & $225 \mathrm{~Hz}$ & Yes & $4 \times$ \\
\hline TIEL2012 [26] & SSHI & N.A. & $\begin{array}{c}\text { T120-A4E } \\
\text { Piezo }\end{array}$ & $5.84 \mathrm{~V}$ & $33.47 \mathrm{nF}$ & $30 \mathrm{~Hz}$ & Yes & $2 \times$ \\
\hline JSSC2012 [27] & PSCE & $5.8 \mu \mathrm{W}$ & Mide V22B & $12.6 \mathrm{~V}$ & $19.5 \mathrm{nF}$ & $174 \mathrm{~Hz}$ & Yes & $1.23 \times$ \\
\hline JSSC2014 [18] & MS-SECE & $\geq 1 \mu \mathrm{W}$ & Murata & $40 \mathrm{~V}$ & $23 \mathrm{nF}$ & $100 \mathrm{~Hz}$ & Yes & N.A. \\
\hline JSSC2014 [28] & $\begin{array}{c}\text { Energy- } \\
\text { investing }\end{array}$ & $0.63 \mu \mathrm{W}$ & Mide V22B & $2.6 \mathrm{~V}$ & $15 \mathrm{nF}$ & $143 \mathrm{~Hz}$ & Yes & $3.6 \times$ \\
\hline TPEL2015 [17] & SSHI & $20 \mu \mathrm{W}$ & Mide V22B & $3.28 \mathrm{~V}$ & $18 \mathrm{nF}$ & $225 \mathrm{~Hz}$ & Yes & $4.5 \times$ \\
\hline TPEL2016 [29] & SECE & $0.43 \mu \mathrm{W}$ & $\begin{array}{c}\text { Q220- } \\
\text { A4303YB }\end{array}$ & $2 \mathrm{~V}$ & $52 \mathrm{nF}$ & $60 \mathrm{~Hz}$ & Yes & $3 \times$ \\
\hline This work & $\begin{array}{c}\text { Connection } \\
\text { switching }\end{array}$ & $0.5 \mu \mathrm{W}$ & Mide V22W & $2.5 \mathrm{~V}$ & $115 \mathrm{nF}$ & $82 \mathrm{~Hz}$ & No & $4.5 \times$ \\
\hline \hline
\end{tabular}

capacitor. The theoretical output power of both parallel and series models are calculated and compared in order to find the condition to switch between the two connection types.

The proposed circuit facilitates transferring energy from the piezoelectric material to the storage capacitor at lower excitation amplitudes and it can maintain at high energy conversion efficiency over a wide range of excitation levels. This shows its strong suitability to real world vibration, where the excitation amplitude varies unpredictably. As opposed to other high-performance synchronized switch interface circuits, such as SSHI or SECE, the proposed circuit does not introduce current pulses to invert or extract charge from PTs. Hence, the performance is less affected from synchronized switch damping, especially when highly-coupled PTs are employed. Furthermore, the inductorless design enables a fully CMOS integrated implementation, which enables a reduction in overall system volume, especially for compact systems such as MEMS energy harvesters.

\section{REFERENCES}

[1] M. Zielinski, F. Mieyeville, D. Navarro, and O. Bareille, "A low power wireless sensor node with vibration sensing and energy harvesting capability," in Computer Science and Information Systems (FedCSIS), 2014 Federated Conference on, 2014, Conference Proceedings, pp. $1065-1071$.

[2] S. P. Beeby and D. Zhu, "Vibration energy harvesting: fabrication, miniaturisation and applications," vol. 9517, 2015, Conference Proceedings, pp. 951 703-951 703-8, 10.1117/12.2179783.

[3] B. S. Srujana, Neha, P. Mathews, and V. P. Harigovindan, "Multi-source energy harvesting system for underwater wireless sensor networks," Procedia Computer Science, vol. 46, no. 0, pp. 1041-1048, 2015.

[4] L. Larcher, S. Roy, D. Mallick, P. Podder, M. de Vittorio, T. Todaro, F. Guido, A. Bertacchini, R. Hinchet, J. Keraudy, and G. Ardila, Vibrational Energy Harvesting. John Wiley Sons, Inc., 2014, pp. 89134.

[5] B. H. Calhoun, D. C. Daly, N. Verma, D. F. Finchelstein, D. D. Wentzloff, A. Wang, S. Cho, and A. P. Chandrakasan, "Design considerations for ultra-low energy wireless microsensor nodes," Computers, IEEE Transactions on, vol. 54, no. 6, pp. 727-740, 2005.

[6] R. Elfrink, V. Pop, D. Hohlfeld, T. M. Kamel, S. Matova, C. de Nooijer, M. Jambunathan, M. Goedbloed, L. Caballero, M. Renaud, J. Penders, and R. van Schaijk, "First autonomous wireless sensor node powered by a vacuum-packaged piezoelectric mems energy harvester," in Electron Devices Meeting (IEDM), 2009 IEEE International, Conference Proceedings, pp. 1-4.
[7] Y. Yang, X.-J. Wei, and J. Liu, "Suitability of a thermoelectric power generator for implantable medical electronic devices," Journal of Physics D: Applied Physics, vol. 40, no. 18, p. 5790, 2007.

[8] M. D. Seeman, S. R. Sanders, and J. M. Rabaey, "An ultra-low-power power management ic for wireless sensor nodes," in Custom Integrated Circuits Conference, 2007. CICC '07. IEEE, Conference Proceedings, pp. $567-570$.

[9] P. D. Mitcheson, E. M. Yeatman, G. K. Rao, A. S. Holmes, and T. C. Green, "Energy harvesting from human and machine motion for wireless electronic devices," Proceedings of the IEEE, vol. 96, no. 9, pp. 14571486, 2008.

[10] S. Priya and D. J. Inman, Energy harvesting technologies. Springer, 2009, vol. 21.

[11] D. Briand, E. Yeatman, S. Roundy, O. Brand, G. K. Fedder, C. Hierold, J. G. Korvink, and O. Tabata, Micro Energy Harvesting. John Wiley Sons, 2015, vol. 12 .

[12] N. Elvin and A. Erturk, Advances in energy harvesting methods. Springer Science \& Business Media, 2013.

[13] L. Bu, H. Xu, and B. Xu, "A novel electrostatic vibration energy harvester array using sidewall electric field," in Solid-State and Integrated Circuit Technology (ICSICT), 2014 12th IEEE International Conference on, 2014, Conference Proceedings, pp. 1-3.

[14] M. Han, Q. Yuan, X. Sun, and H. Zhang, "Design and fabrication of integrated magnetic mems energy harvester for low frequency applications," Microelectromechanical Systems, Journal of, vol. 23, no. 1, pp. 204-212, 2014

[15] A. Badel, D. Guyomar, E. Lefeuvre, and C. Richard, "Efficiency enhancement of a piezoelectric energy harvesting device in pulsed operation by synchronous charge inversion," Journal of Intelligent Material Systems and Structures, vol. 16, no. 10, pp. 889-901, 2005.

[16] Y. K. Ramadass and A. P. Chandrakasan, "An efficient piezoelectric energy harvesting interface circuit using a bias-flip rectifier and shared inductor," Solid-State Circuits, IEEE Journal of, vol. 45, no. 1, pp. 189204, 2010.

[17] L. Shaohua and F. Boussaid, "A highly efficient p-sshi rectifier for piezoelectric energy harvesting," Power Electronics, IEEE Transactions on, vol. 30 , no. 10 , pp. 5364-5369, 2015.

[18] P. Gasnier, J. Willemin, S. Boisseau, G. Despesse, C. Condemine, G. Gouvernet, and J. J. Chaillout, "An autonomous piezoelectric energy harvesting ic based on a synchronous multi-shot technique," Solid-State Circuits, IEEE Journal of, vol. 49, no. 7, pp. 1561-1570, 2014.

[19] A. Badel, G. Sebald, D. Guyomar, M. Lallart, E. Lefeuvre, C. Richard, and J. Qiu, "Piezoelectric vibration control by synchronized switching on adaptive voltage sources: Towards wideband semi-active damping," The Journal of the Acoustical Society of America, vol. 119, no. 5, pp. $2815-2825,2006$

[20] H. Ji, J. Qiu, L. Cheng, and H. Nie, "Semi-active vibration control based on unsymmetrical synchronized switch damping: Analysis and experimental validation of control performance," Journal of Sound and Vibration, vol. 370, pp. 1-22, 2016.

[21] D. Guyomar, A. Faiz, L. Petit, and C. Richard, "Wave reflection and transmission reduction using a piezoelectric semipassive nonlinear 
technique," The Journal of the Acoustical Society of America, vol. 119, no. 1, pp. 285-298, 2006.

[22] M. Dini, A. Romani, M. Filippi, V. Bottarel, G. Ricotti, and M. Tartagni, "A nanocurrent power management ic for multiple heterogeneous energy harvesting sources," Power Electronics, IEEE Transactions on, vol. 30, no. 10 , pp. 5665-5680, 2015.

[23] T. T. Le, H. Jifeng, A. v. Jouanne, K. Mayaram, and T. S. Fiez, "Piezoelectric micro-power generation interface circuits," IEEE Journal of Solid-State Circuits, vol. 41, no. 6, pp. 1411-1420, 2006

[24] V. W. Ng and S. R. Sanders, "A high-efficiency wide-input-voltage range switched capacitor point-of-load dc-dc converter," IEEE Transactions on Power Electronics, vol. 28, no. 9, pp. 4335-4341, 2013.

[25] L. C. J. Blystad, E. Halvorsen, and S. Husa, "Piezoelectric mems energy harvesting systems driven by harmonic and random vibrations," Ultrasonics, Ferroelectrics, and Frequency Control, IEEE Transactions on, vol. 57, no. 4, pp. 908-919, 2010.

[26] J. Liang and W.-H. Liao, "Improved design and analysis of self-powered synchronized switch interface circuit for piezoelectric energy harvesting systems," Industrial Electronics, IEEE Transactions on, vol. 59, no. 4, pp. 1950-1960, 2012.

[27] T. Hehn, F. Hagedorn, D. Maurath, D. Marinkovic, I. Kuehne, A. Frey, and Y. Manoli, "A fully autonomous integrated interface circuit for piezoelectric harvesters," IEEE Journal of Solid-State Circuits, vol. 47 , no. 9, pp. 2185-2198, 2012.

[28] D. Kwon and G. A. Rincon-Mora, "A single-inductor 0.35 um cmos energy-investing piezoelectric harvester," Solid-State Circuits, IEEE Journal of, vol. 49, no. 10, pp. 2277-2291, 2014.

[29] M. Dini, A. Romani, M. Filippi, and M. Tartagni, "A nanopower synchronous charge extractor ic for low-voltage piezoelectric energy harvesting with residual charge inversion," IEEE Transactions on Power Electronics, vol. 31, no. 2, pp. 1263-1274, 2016.

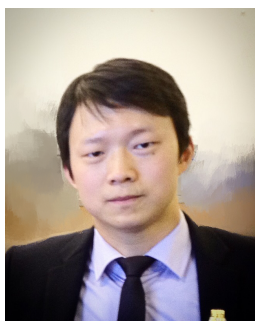

Sijun Du (S'14) received the B.Eng. degree from University Pierre and Marie Curie, Paris, France, in 2011 and the M.Sc. degree in Electrical Electronics Engineering from Imperial College, London, U.K., in 2012 .

He is currently working towards the Ph.D. Degree at the Nanoscience Centre, Department of Engineering at the University of Cambridge, U.K. His research interests include macroscopic and MEMS (Microelectromechanical Systems) energy harvesters, associated interface circuits, power management circuits, DC-DC converters and rectification circuits.

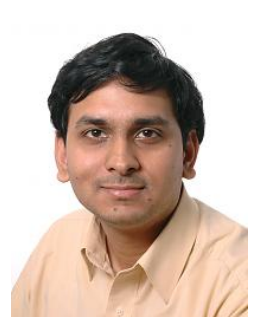

Ashwin A. Seshia (S'98-M'02-SM'10) received the B.Tech. degree in engineering physics from IIT Bombay, in 1996, and the M.S. and Ph.D. degrees in electrical engineering and computer sciences from the University of California, Berkeley, in 1999 and 2002, respectively. He joined the Engineering Department at the University of Cambridge, in 2002, where he is currently a Reader of Microsystems Technology and a Fellow of Queens College. His research interests include mi- crofabricated devices and integrated microsystems, vibratory and resonant phenomena at the MEMS-scale, acoustic wave devices, resonators and oscillators, time and frequency metrology, transducers and interfaces, physical measurements at small length scales, sensors and sensor systems, and vibration energy harvesting.

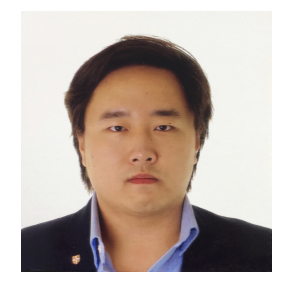

Yu Jia (S'10-M'14) is currently a Lecturer in Mechanical Engineering at the University of Chester, and leads the Smart Microsystems Research Group. Yu Jia received a First Class (Honours) degree in MEng Electromechanical Engineering from the University of Southampton in 2010, and $\mathrm{PhD}$ in Engineering from the University of Cambridge in 2014. He was then a Research Associate at Cambridge for a year. $\mathrm{He}$ is the co-founder of 8power Ltd. $\mathrm{He}$ is also a steering board member of the Energy Harvesting Network. His research interests involve vibration energy harvesting, micro-electromechanical systems, nonlinear vibration dynamics and smart integrated systems. 